\title{
Recumbent folding in ice sheets: a core-referential study
}

\author{
H. PAul JACOBSON, Edwin D. WADDINGTON \\ Department of Earth and Space Sciences, University of Washington, Box 351310, Seattle, Washington 98195-1310, U.S.A. \\ E-mail:edw@ess.washington.edu
}

\begin{abstract}
To better understand apparent stratigraphic disturbances in ice cores such as Greenland Ice Sheet Project 2 (GISP2), we examine how ice-sheet flow can transform gentle open folds into order-disturbing recumbent folds. The initial disturbances in the stratigraphy have their roots in transient dynamic processes and local rheological inhomogeneities, but the kinematics of even a simple ice-flow model can deform these disturbances enough to alter paleoclimatic interpretation of an ice core. The local vorticity number suggests which structures can be passively overturned, but analyzing the finite strain along particle paths gives a more complete picture, especially when taken relative to a hypothetical core location. Core-relative isochrones, or "pre-cores", predict which stratigraphic disturbances will appear as obviously overturned layers in a core. The deformation-gradient tensor along particle paths allows us to calculate the rotation of segments of various reference slopes. These calculations suggest that observed $20^{\circ}$ dips in the GISP2 core are rotating on a time-scale of a few hundred years and could result from distortions with much smaller slopes produced upstream. The time during which they can be recognized to be overturning is short because the rotation rate is high. Once overturned they are flattened further and may be hard to recognize, especially in the small cross-section of a core.
\end{abstract}

\section{NOTATION}

$\dot{b}$

$b \quad$ Accumulation rate

$B(x) \quad$ Bed profile $(B(x)=0)$

$C \quad x$ coordinate of a hypothetical ice core

$\hat{d} \quad=(S-z) / h$, normalized depth

$\mathrm{d} \mathbf{X}, \mathrm{d} \mathbf{x} \quad$ Reference and current segments

F Deformation-gradient tensor

$\mathbf{F}_{\langle\mathbf{X x}\rangle} \quad \mathbf{F}$ with $\mathbf{X}$ reference point, $\mathbf{x}$ current point

$F_{x x} \quad$ One of the components of $\mathbf{F}$

$H \quad$ Thickness at the divide

$h(x) \quad=S-B$, thickness

L Velocity-gradient tensor

$L \quad$ Length of flowband, from divide to terminus

$Q(x) \quad$ Flux through flowband cross-section at $x$

$S^{\prime} \quad$ Surface slope, gradient of $S(x)$

$S(x) \quad$ Surface profile

$T \quad=H / \dot{b}$, time-scale

$\hat{u}(\hat{d}) \quad$ Vertical profile of $u(x, z)$

$\bar{u}(x) \quad$ Mean horizontal velocity

$u(x, z) \quad$ Horizontal-velocity component

$v \quad=0$, transverse-velocity component

$\hat{w}(\hat{d}) \quad$ Vertically integrated $\hat{u}$

$w(x, z) \quad$ Vertical-velocity component

$W_{k} \quad$ Kinematic vorticity number

$x \quad$ Horizontal coordinate (downstream)

$y \quad$ Coordinate orthogonal to $x$ and $z$

$z \quad$ Vertical coordinate

$\Theta, \theta \quad$ Reference and current segment angles

$\dot{\theta} \quad$ Rotation rate, time derivative of $\theta$

$\theta_{f} \quad$ Pre-core slope angle

$\theta_{r} \quad$ Rate-based critical wrinkle angle

$\partial_{x} u \quad=\partial u / \partial x$, one of the terms of $\mathbf{L}$

\section{INTRODUGTION}

Underlying the paleoclimatic interpretation of ice cores is the assumption that a core samples the ice in the order in which it was deposited. Some post-depositional modification in the ice stratigraphy, such as thinning of annual layers, is anticipated and can be included in the interpretation of the climatic signal. Unanticipated thinning would introduce errors in some climatic signals, such as the accumulation rate, but actual alteration in the order of some of the stratigraphic layers in the core sample affects the entire climatic signal they carry.

\subsection{Evidence from ice caps}

The suggestion that the Greenland Icecore Project (GRIP) ice core showed evidence of major climatic shifts in part of the Eemian interglacial period (GRIP Members, 1993) proved to be particularly controversial when the nearby Greenland Ice Sheet Project 2 (GISP2) core lacked the corresponding oxygen-isotope oscillations (Grootes and others, 1993). It was suggested that one or both of the cores had been altered by folding or other forms of mixing. Further research found evidence for differential thinning, and possibly even folding, in the lower $10 \%$ of the ice by comparing the water isotope signal with the atmospheric dissolved gases signal (Fuchs and Leuenberger, 1996). A detailed examination of visible stratigraphy showed dips of up to $20^{\circ}$, and even a few small overturned folds in the lower $25 \%$ of the cores (Alley and others, 1995). A small-scale structure, called "stripes", was also identified in the GISP2 core (Alley and others, 1997). Photographs of these stripes, and small folds associated with them, can be seen in Alley and others (1997). The difficulty in identifying internal 
radar layers in deep ice may sometimes be an indication of stratigraphic disturbance at these depths (Robin, 1983).

Interaction of contrasting layers has been been proposed as a cause of such folding (Dahl-Jensen and others, 1997). Layering in anisotropic ice directly under the divide, where it is subject to vertical compression, appears, in theory, to be potentially unstable (Azuma and Goto-Azuma, 1996; Castelnau and others, 1998; Thorsteinsson and Waddington, 2002). Recumbent folding observed at the terminus of Barnes Ice Cap, Canadian Arctic, has been attributed to advancing or retreating margins (Hudleston, 1977). Foliation developed under one flow pattern could be passively deformed and folded when subjected to a different flow.

The GRIP core was drilled close to the current summit divide of the Greenland ice sheet in the hope of observing a maximum possible thickness of undisturbed ice. The GISP2 core was placed ten ice thicknesses $(30 \mathrm{~km})$ to the west of the divide to give a complementary sample of nearby flank-flow ice. But if the divide moved around during the last glacial cycle (Anandakrishan and others, 1994; Marshall and Cuffey, 2000) it may be best to consider both locations as neardivide sites. Other cores, such as Dye 3 (Greenland), Camp Century (Greenland) and Byrd (Antarctica) have been drilled on clearly flank positions.

Ice-penetrating radar can follow the internal stratigraphy over large horizontal extents, to complement the very small-scale details seen in ice cores. Examples can be found from Greenland (Jacobel and Hodge, 1995), East Antarctica (Robin, 1983) and Siple Dome, West Antarctica (Nereson and others, 1998). Unfortunately, the ability of radar to image folds at scales smaller than tens to hundreds of meters may be limited. These intermediate-scale folds are also difficult to clearly identify in ice cores. Without azimuthal orientation information or reliable "up" indicators (Alley and others, 1995) it is difficult to say whether a dip in the stratigraphy in a core sample is the start of a fold, or the overturned limb of a well-developed fold.

\subsection{A passive-shearing model of folding}

To understand how stratigraphic layers in an ice sheet could be reordered, Waddington and others (2001) (also Waddington and others, 1995; Jacobson and Waddington, 1996; Jacobson, 2001) have studied the kinematics of forming recumbent folds from gentle disturbances in otherwise steady-state stratigraphy. At scales of decimeters to decameters, these initial disturbances could have their roots in some transient dynamic process, most likely involving local rheological inhomogeneities. Waddington and others (2001) and Thorsteinsson and Waddington (2002) evaluate in more detail some processes that could possibly generate such small-scale wrinkles. At the scale of hundreds of meters, the arches created in internal layers by the special flow pattern found under an ice divide (Raymond, 1983) can be a source of initial layer disturbance if a stationary ice divide subsequently migrates rapidly. These arches have been observed in Antarctica at Fletcher Promontory (Vaughan and others, 1999), Siple Dome (Nereson and others, 1998) and Roosevelt Island (Conway and others, 1999). In a separate paper (unpublished manuscript by Jacobson and Waddington) we investigate the potential for generating folds from these arches. However, we do not have a comprehensive theory of how and where injection of disturbances might occur at other places and at other spatial scales, and so we will limit

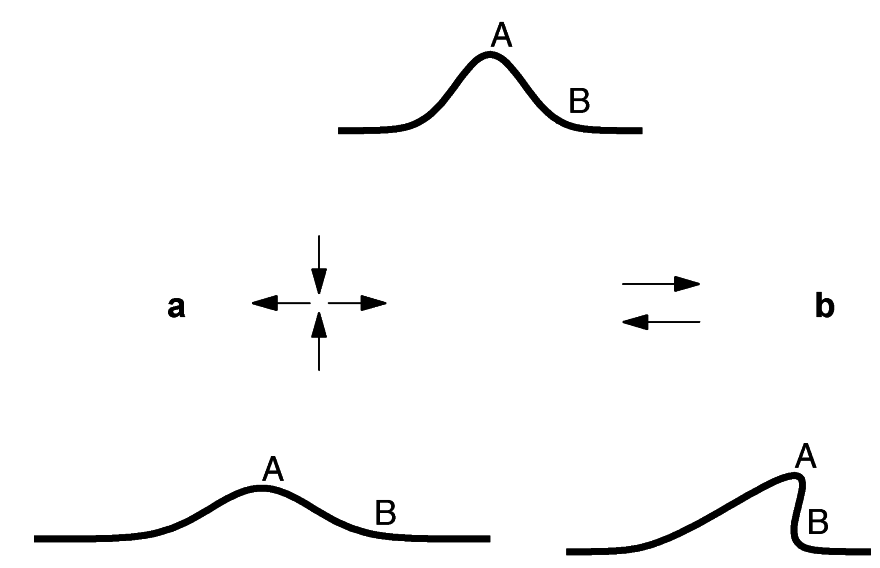

Fig. 1. The deformation of a disturbed layer under (a) pure shear and (b) simple shear. Pure shear, with vertical compression, flattens the disturbance. Simple shear steepens (and overturns) the $A-B$ limb while leaving the disturbance amplitude unchanged.

this study to examining the consequences of injection at a variety of positions.

The deformation in an ice sheet in plane strain near its center can be represented as a combination of vertically compressive pure shear and bed-parallel simple shear. The pure shear thins and stretches the stratigraphic layers. It also flattens disturbances in these layers. The simple shear, on the other hand, "catches" these wrinkles and deforms them into order-disrupting recumbent folds. These two effects are illustrated in Figure 1, where the contrasting rotations of the $\mathrm{A}-\mathrm{B}$ limb are highlighted. When we discuss the angle and rotation of a segment, it is the behavior of just such a portion of a disturbance that we have in mind.

Waddington and others (2001) used this trade-off between stretching and shearing to determine where disturbances of any given slope would be flattening and where they would be overturning. They assessed the plausibility of several proposed disturbance sources, and applied their stability limit estimates to the Dye 3, GRIP, GISP2 and Siple Dome ice-core sites. Their simple approach was based entirely on the strain rate at a point (e.g. the point at which a disturbance might be created by localized inhomogeneous flow). As they noted, their assessment gave an optimistic view of stratigraphic integrity, because it neglected variations in strain rate experienced by disturbances as they moved (a) over undulating bedrock, and (b) into deeper regions with stronger bed-parallel shear strain rates. They were able to estimate the time required for disturbances to overturn, and the distance that disturbances would move during this time, but only for disturbances that were already rotating strongly at the point of injection. They suggested that finite-strain calculations, following disturbances as they moved along particle paths, would produce better assessments of the behavior of marginally unstable disturbances, and would greatly advance understanding of this passive-folding process. This paper analyzes those finite strains to understand features of folding that could not be addressed by Waddington and others (2001).

One measure of the mixture of pure and simple shear is the kinematic vorticity number, $W_{\mathrm{k}}=\overline{\dot{\omega}} / \overline{\dot{\epsilon}}$ (Means and others, 1980). This is defined so that $W_{\mathrm{k}}=0$ for pure shear, and $W_{\mathrm{k}}=1$ for simple shear. $\overline{\dot{\omega}}$ is the rotation rate of the principal axes, and $\dot{\dot{\epsilon}}$ is the difference in their strain rates, $\dot{\epsilon}_{\text {max }}-\dot{\epsilon}_{\text {min }}$. In Hudleston and Hooke (1980) this is called 


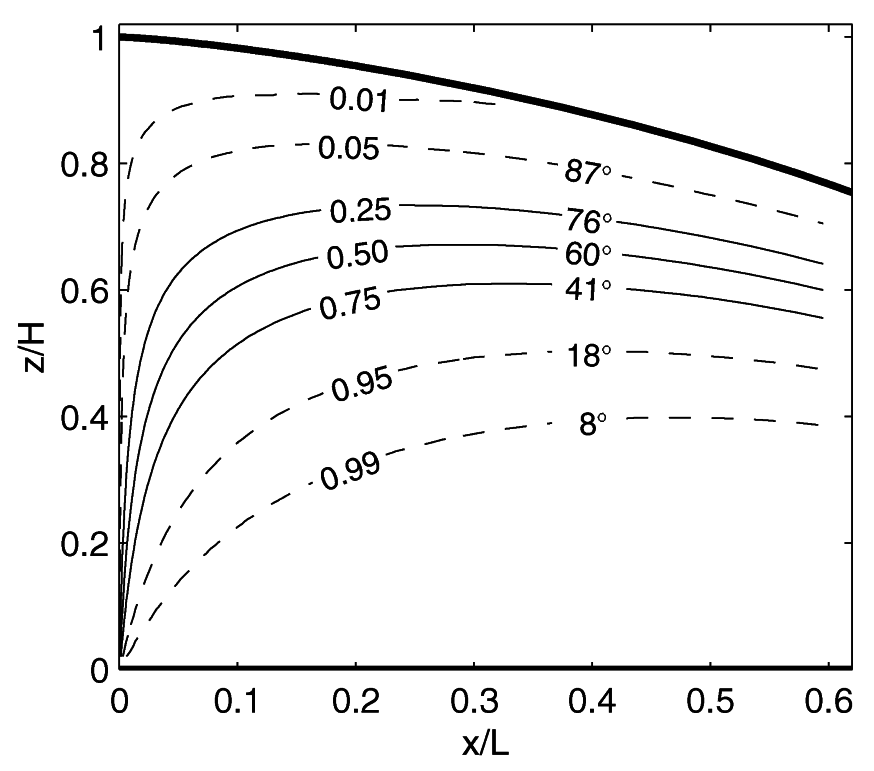

Fig. 2. The kinematic vorticity number, $W_{\mathrm{k}}$, and the critical wrinkle angle, $\theta_{\mathrm{r}}$, for a simple ice-sheet model. $z / H$ describes the relative height in the ice sheet; $x / L$ the distance from the ice-sheet center. The lefthand number on each contour, $W_{\mathrm{k}}$, is a measure of the relative magnitudes of pure and simple shear. Pure shear dominates near the surface and under the divide $\left(W_{\mathrm{k}}\right.$ close to 0$)$ while simple shear dominates elsewhere ( $W_{\mathrm{k}}$ close to 1$)$. The righthand number, $\theta_{\mathrm{r}}$, is the segment angle that is not rotating at this point in the flow $\left(\cos ^{-1} W_{\mathrm{k}}\right)$.

the index of simple shear, $I_{\mathrm{ss}}$. Under pure shear, the principal axes do not rotate, while under simple shear, their rotation rate equals the strain rate.

The vorticity number ${ }^{1}$ for a simple ice-sheet flowband model is shown in Figure 2. Near the surface of the ice sheet, vertical compression dominates, so $W_{\mathrm{k}}$ is close to 0 . This is also true directly under the divide where the horizontal flow is negligible. On the flank, the proportion of simple shear increases with depth, and $W_{\mathrm{k}}$ approaches unity.

In pure shear (as in Fig. la), vertical and horizontal segments do not rotate, while segments on either side of vertical rotate toward horizontal. Under simple shear, all segments, except those parallel to the plane of shear, rotate in the same direction (clockwise in Fig. 1b). In mixed pure and simple shear $\left(0<W_{\mathrm{k}}<1\right)$, there is some angle, $\theta_{\mathrm{r}} \approx \cos ^{-1} W_{\mathrm{k}}$, between vertical and horizontal that is not rotating, and which separates the clockwise rotating segments from the anticlockwise ones (Bobyarchick, 1986). The contours in Figure 2 have been labeled with this angle. The critical wrinkle segment slope, $m_{\text {crit }} \approx S^{-1}$, in Waddington and others (2001) is approximately the tangent of $\theta_{\mathrm{r}}$, and their dimensionless shear number, $S$, is a variant on $W_{\mathrm{k}}$.

The wrinkle in Figure 1 with the A-B segment at about $41^{\circ}$ would be flattening if it occurred above the $41^{\circ}$ contour in Figure 2, but would be steepening (rotating clockwise) if it was below this contour.

$\theta_{\mathrm{r}}$ is not simply a divider between segments that rotate one way versus the other. As a segment moves along a particle path, $W_{\mathrm{k}}$ increases and $\theta_{\mathrm{r}}$ decreases. The effect on the segment's rotation can be seen by considering a segment

${ }^{1}$ Our model geometry and notation are explained in section 2.2. with an angle equal to $\theta_{\mathrm{r}}$ at time $t$ on the path. At this point it is not rotating. At $t+\mathrm{d} t$, a short time later, it will have practically the same angle, but will be further along the path, where $\theta_{\mathrm{r}}$ is smaller. Since it is now steeper than $\theta_{\mathrm{r}}$, its rotation is clockwise, away from $\theta_{\mathrm{r}}$. Conversely, at an earlier time, $t-\mathrm{d} t$, the segment angle would have been larger than the local $\theta_{\mathrm{r}}$, which means that the segment was rotating anticlockwise, toward $\theta_{\mathrm{r}}$. In effect, the $\theta_{\mathrm{r}}$ angle is a turnaround point. A segment can flatten until it reaches the local $\theta_{\mathrm{r}}$, at which point it reverses rotation direction and starts to steepen.

\subsection{Observation points}

Since segments can change their direction of rotation, we need to look at the evolution of a disturbance over a finite interval along a path in order to gain a more detailed understanding of the folding process than that which the first analysis of $\theta_{\mathrm{r}}$ by Waddington and others (2001) provides. This requires choosing meaningful end-points for such an interval.

The obvious choice for the starting point is where the disturbance originated, assuming, of course, that there is a time when the transient dynamics generating the disturbance cease to be significant and we can focus on the kinematics. In this paper, we do not examine the complexities of this transition. In a separate paper (unpublished manuscript by Jacobson and Waddington) we investigate one such transition to kinematic overturning, which occurs in the special flow field found under an ice divide (Raymond, 1983). Here we limit our study to following smaller-scale disturbances, injected at a variety of positions, after this transition has occurred.

We have found it equally productive to specify the interval end-point, as the place where we find an observed or hypothetical fold, and ask what sort of disturbance might have been its precursor. In particular, we look at a vertical set of observation points, such as might be sampled in an ice core.

To study this kinematic folding, we use a flowband model to calculate the ice velocity and its gradient at all points, and use these to calculate particle paths. Then we define core-relative isochrones and show their relevance to folding. Next we calculate the deformation gradient and show how it gives more general information on segment rotation. Finally, we ask how much information is needed to predict where observed folds might have originated. While the best evidence for folding has been found in the lower $20 \%$ of ice sheets, and while models predict the greatest likelihood of folding near the bed, nevertheless our models show that folds can be encountered virtually anywhere in an ice sheet, except close to the surface, or directly under a stable ice divide.

\section{FLOWBAND MODEL}

After defining some terminology for disturbances and angles, we will describe a simple flowband model of an ice sheet. This model calculates the velocity at points in the flowband as a function of position and model geometry. Using this velocity field, we can calculate the velocity gradient, $W_{\mathrm{k}}$, and $\theta_{\mathrm{r}}$ at specific points. We can also calculate particle paths and finite strain along these paths. 


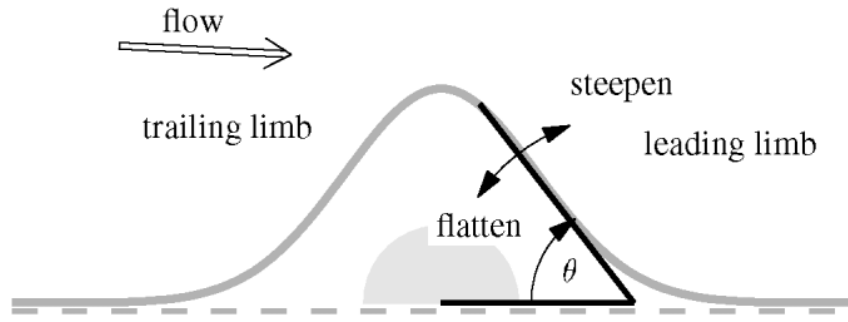

Fig. 3. Prototypical disturbance, illustrating our notation, including leading limb, trailing limb and $\theta$. Segment slope is measured relative to horizontal (pointing upstream). Not shown is the slight slope (relative to horizontal) of the undisturbed stratigraphy. One could imagine a disturbance such as this forming around a rheologically stiffer "lump" in the ice. Such a lump would also disturb the layers below it.

\subsection{Disturbance notation}

A prototypical upward disturbance with key terms is depicted in Figure 3. Such a wrinkle might be the result of stratigraphic layers draping around a transient rheologically harder lump of ice (Waddington and others, 2001). Of particular interest is the leading limb (the A-B edge in Fig. 1), which is the portion of this disturbance which would overturn when sheared in the direction of flow. We will also refer to the trailing limb, the portion that will flatten under both pure and simple shear. If the disturbance were inverted (that is, a dip) the limb on the upstream side of the disturbance would be the limb at risk of overturning. The required adjustment in terminology is left to the reader.

We will use $\theta$ for the angle of a segment, measuring it relative to the horizontal axis pointing in the upstream direction (to the left in our prototype). This choice of angle orientation means that the initial angle of a leading limb will be in the $0-90^{\circ}$ range, and will increase to $90^{\circ}$ and beyond when the leading limb overturns. A trailing limb will start in the $90-180^{\circ}$ range and rotate toward $180^{\circ}$.

For the purposes of this paper, we ignore the distinction between horizontal and the slope of undisturbed stratigraphy. Near the center of an ice sheet, the slope of stratigraphy over a flat bed is negligible, on the order of $0.5^{\circ}$ or less.

\subsection{Velocity model}

We use a simple flowband model, with enough detail to capture the change in the vorticity number along particle paths but without complicating details. This model assumes that surface slopes are small and that the bed is frozen. This is true for the central portion of many, though not all, ice sheets and ice caps. Allowing sliding on all or portions of the base would require a significantly more complicated flowband model; however, as long as some ice flow due to horizontal shear is present, folding as encapsulated in Figure 1 will be present.

Figure 4 illustrates our model. The coordinate system is aligned with the direction of flow. The horizontal coordinate in this direction is $x$, while $z$ is the vertical coordinate. The corresponding velocity components are $u$ and $w$ (positive upward). Perpendicular to these is $y$, with its velocity component $v$, being zero by definition.

The geometric inputs to our velocity model are the surface profile $S(x)$ and the bed profile $B(x)$. The flowband ice equivalent thickness is $h(x)=S-B$.

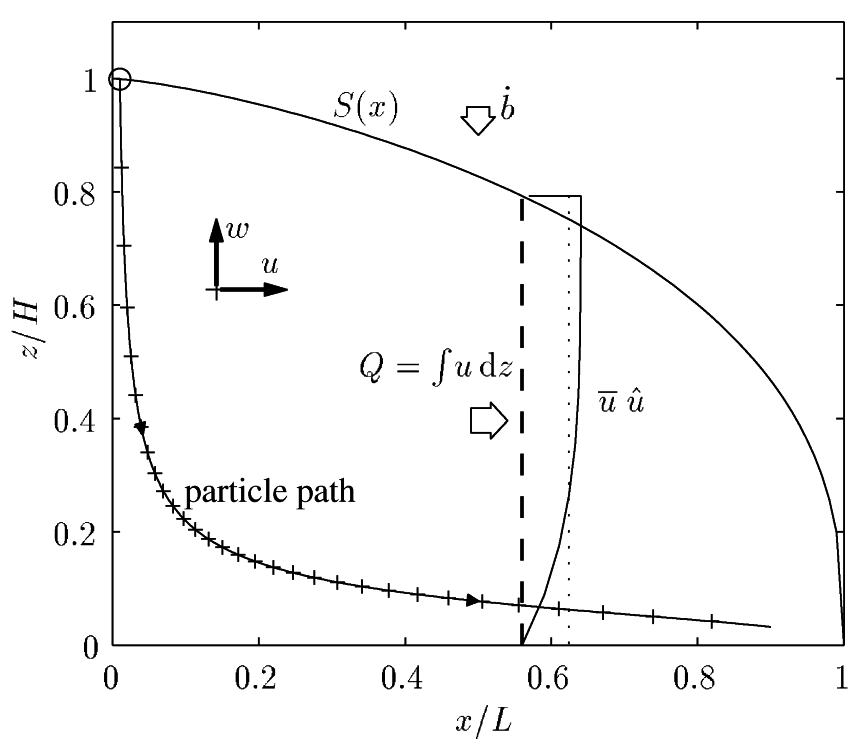

Fig. 4. Flowband geometry and notation defined in text. Crosses on the particle path mark equal elapsed time intervals.

The transverse geometry can be expressed in terms of a relative flowband width, and used to calculate the transverse spreading rate $\partial_{y} v$, as a function of $u$ and $x$.

We also specify the flux $Q(x)$, through the flowband cross-section at $x$. Dividing by the thickness gives a depthaveraged horizontal velocity, $\bar{u}(x)=Q / h$.

Rounding out the inputs is an expression for the vertical profile (shape function) of the horizontal velocity, $\hat{u}(x, z)$. The source of this function is a dynamic model that includes momentum conservation and ice rheology. The horizontal velocity can then be written as

$$
u(x, z)=\bar{u}(x) \hat{u}(x, z) .
$$

Since the integral of $u$ over the thickness equals the flux, the integral of $\hat{u}$ over $z$ must equal $h$. The vertical velocity can be derived from $u(x, z)$ by incompressibility,

$$
w(x, z)=-\int_{B}^{z}\left(\partial_{x} u+\partial_{y} v\right) \mathrm{d} z+w(x, B) .
$$

We simplify the calculations with a number of assumptions, which can be selectively relaxed to explore their effect on the results. Few are essential to this analysis, but they allow us to keep the description simple, while retaining the features of the flow that are key to folding. The resulting model was first proposed by Vialov (1958) (see also Reeh, 1988).

We assume that the base is flat, $B(x)=0$, and that the ice is frozen to the bed, so that $u(x, B)=w(x, B)=0$. The flowband width is uniform, so that all the gradient terms in the $y$ direction vanish. This approximates the flow on an ice ridge or a highly elongated dome. The velocity gradient is then

$$
\mathbf{L}=\nabla \mathbf{v}=\left[\begin{array}{cc}
\partial_{x} u & \partial_{z} u \\
\partial_{x} w & \partial_{z} w
\end{array}\right]
$$

We assume that the ice-sheet surface is in steady state. The flux $Q(x)$ through a cross-section must equal the integrated accumulation upstream. If we also assume that the accumulation rate, $\dot{b}$, is uniform in space, then the flux is $Q(x)=\dot{b} x$.

This uniform-accumulation assumption requires that we specify the length or span of the flowband $L$, in order to determine the model geometry. In effect, we specify the 
location of a calving front that can handle any flux. The conditions at such a terminus are not realistic, but they do not adversely affect the model a short distance inland. $L$ could also be thought of as a virtual or effective length. If an ice sheet terminates in an ice stream (as Siple Dome does) or a narrow ablation zone, the terminus profile would be different, but this model would still be useful over a wide region around the divide.

To specify $\hat{u}$ and $S$, we assume that the ice is isothermal, and use the shallow-ice approximation (Hutter, 1983; Paterson, 1994, p. 262) with Glen's flow law. This flow law assumes that the deviatoric stress $\sigma^{\prime}$, and strain rate $\dot{\epsilon}$, are related by $\dot{\epsilon}=A \sigma^{\prime 3}$, where $A$ is a temperature-dependent flow parameter. Since, in a typical ice sheet, the length is much larger than the thickness, the shearing component, $\partial_{z} u$, is the largest term of the velocity gradient over much of the flowband, and the flow law can be written as

$$
\begin{gathered}
\partial_{z} u \approx 2 \dot{\epsilon}_{x z} \approx 2 A \sigma_{x z}^{\prime 3}=-2 A(\rho g)^{3} S^{\prime 3} h^{3} \hat{d}^{3} \\
\hat{d}=\frac{S-z}{h} \text { normalized depth } \\
\sigma_{x z}^{\prime}=-\rho g S^{\prime} h \hat{d} \text { shear stress }
\end{gathered}
$$

where $S^{\prime}$ is the surface gradient. Integrating Equation (4) upward from the bed gives the horizontal velocity and its shape function.

$$
\begin{gathered}
u=-2 A(\rho g)^{3} S^{\prime 3} h^{3} \int_{0}^{z} \hat{d}^{3} \mathrm{~d} z \\
=-\frac{1}{2} A(\rho g)^{3} S^{\prime 3} h^{4}\left(1-\hat{d}^{4}\right)=\bar{u} \hat{u}(\hat{d}) \\
\hat{u}(\hat{d})=\frac{5}{4}\left(1-\hat{d}^{4}\right)
\end{gathered}
$$

In this case, $\hat{u}$ is a function of only $\hat{d}$.

The corresponding vertical velocity (using Equation (2)) is

$$
\begin{gathered}
w=-\dot{b} \hat{w}(\hat{d})+u S^{\prime}(1-\hat{d}) . \\
\hat{w}(\hat{d}) \equiv \int_{\hat{d}}^{1} \hat{u}(\hat{d}) \mathrm{d} \hat{d}=1-\frac{5}{4} \hat{d}+\frac{1}{4} \hat{d}^{5} .
\end{gathered}
$$

A surface profile consistent with this $\hat{u}(\hat{d})$ can be derived from the steady-state expression for the flux.

$$
Q(x)=\dot{b} x=\int_{B}^{S} u(x, z) \mathrm{d} z=-\frac{2}{5} A(\rho g)^{3} S^{\prime 3} h^{5}
$$

This can be rewritten as a differential equation in $S^{\prime}$, which can be solved numerically. In the simple case of a flat bed and uniform accumulation, it can be solved analytically (Vialov, 1958) giving

$$
S(x)=H\left[1-\left(\frac{x}{L}\right)^{4 / 3}\right]^{3 / 8} .
$$

The maximum thickness, $H=h(0)$, is related to the other parameters $(L, \dot{b}$ and $A)$ by

$$
H=\left(\frac{20 \dot{b} L^{4}}{A(\rho g)^{3}}\right)^{1 / 8} .
$$

The horizontal coordinate $x$ scales with $L$, while the vertical coordinate and ice-sheet thickness scale with $H$. With a typical $H / L$ ratio of $1 / 50$, the surface slope $S^{\prime}$ (for $x<0.5 L)$ is small. The time-scale is set by $T=H / \dot{b}$. The velocities, $u$ and $w$, scale with $L / T$ and $H / T(=\dot{b})$ respectively.
The velocity gradient, L (Equation (3)), scales as

$$
\left[\begin{array}{cc}
1 & L / H \\
H / L & 1
\end{array}\right] \frac{1}{T}
$$

Since $L$ is much larger than $H$, the $\partial_{z} u$ term clearly dominates. The $\partial_{x} w$ term is $(H / L)^{2}$ times smaller, and can in many cases be assumed to be zero.

\subsection{Segment rotation rate}

With a velocity model we can calculate the velocity gradient, and from that the rotation rate $\dot{\theta}$, of a segment with an angle of $\theta$.

$$
\dot{\theta}=\partial_{z} u \sin ^{2} \theta-\left(\partial_{x} u-\partial_{z} w\right) \sin \theta \cos \theta-\partial_{x} w \cos ^{2} \theta
$$

When $W_{\mathrm{k}}$ is small (mostly pure shear), the $\left(\partial_{x} u-\partial_{z} w\right)$ term dominates, resulting in rotation away from vertical for most angles. In most of the ice sheet $\partial_{z} u$ dominates, producing a positive $\dot{\theta}$ for most angles except a small set close to $0^{\circ}$ (horizontal upstream). At some points over an uneven bed, it is possible for $\partial_{z} w$ to be sufficiently negative that $\dot{\theta}>0$ for all segment angles. At such points $W_{\mathrm{k}}>1$.

When $\partial_{x} w$ is negligible, the segment is not rotating $(\dot{\theta}=0)$ if $\tan \theta \approx\left(\partial_{x} u-\partial_{z} w\right) / \partial_{z} u$. In this case, the vorticity number can be written as

$$
\begin{aligned}
W_{\mathrm{k}}=\frac{\overline{\dot{\omega}}}{\overline{\dot{\epsilon}}} & =\frac{\partial_{z} u-\partial_{x} w}{\sqrt{\left(\partial_{z} u+\partial_{x} w\right)^{2}+\left(\partial_{x} u-\partial_{z} w\right)^{2}}} \\
& \approx \frac{\partial_{z} u}{\sqrt{\partial_{z} u^{2}+\left(\partial_{x} u-\partial_{z} w\right)^{2}}} \\
& \approx \cos \left(\tan ^{-1} \frac{\partial_{x} u-\partial_{z} w}{\partial_{z} u}\right)=\cos \theta_{\mathrm{r}},
\end{aligned}
$$

confirming the relationship between $W_{\mathrm{k}}$ and $\theta_{\mathrm{r}}$ shown in Figure 2.

In general there are two segment angles that do not rotate, one of which is the reference steady-state stratigraphy angle. They merge into one for simple shear. $\cos ^{-1} W_{\mathrm{k}}$ is the sum of angles of these two non-rotating segments. For small $\partial_{x} w$, the approximation in Expression (16) is valid because one of these is practically horizontal. Waddington and others (2001) refine this idea of a non-rotating segment by calculating the segment that is not rotating relative to the reference steady-state isochrone at a point. However, this requires knowing the slope and rotation rate of the isochrones, which must be calculated from particle paths.

\subsection{Particle paths}

We calculate particle paths by solving the pair of differential equations:

$$
\dot{x}=u(x, z) \quad \dot{z}=w(x, z) .
$$

Path calculation can start at any point in the ice sheet, and run either forward or back in time. Each point along a path is defined by a triplet of values, $[x, z, t]$. For a steady-state geometry, only relative times are important. We solve these differential equations numerically with the ordinary differential equation (ODE) routines provided with MATLAB (Shampine and Reichelt, 1997).

In velocity and particle-path calculations, the horizontal and vertical coordinates can be rescaled independently, but the slope and finite deformation calculations (next section) 
Table 1. Characteristic geometry parameters

\begin{tabular}{lcc}
\hline Parameter & Siple Dome & Greenland \\
\hline$L(\mathrm{~km})$ & 50 & 300 \\
$H(\mathrm{~m})$ & 1000 & 3000 \\
$H / L$ & $1 / 50$ & $1 / 100$ \\
$\dot{b}\left(\mathrm{~m} \mathrm{a}^{-1}\right)$ & 0.1 & 0.3 \\
$T=H / \dot{b}$ (years $)$ & 10000 & 10000 \\
$A\left(\mathrm{kPa}^{-3} \mathrm{~s}^{-1}\right)$ & $5.6 \times 10^{-16}$ & $3.3 \times 10^{-16}$ \\
\hline
\end{tabular}

retain a dependence on the $H / L$ ratio. For most of our examples, we use an ice ridge with dimensions comparable to Siple Dome, which has a 1/50 ratio. We also examine a Greenland-like ridge which has a 1/100 ratio (Table 1).

\section{PRE-CORES}

A conventional isochrone is the set of glacial ice of the same age, where age is the time since the ice accumulated at the surface. We can equally well construct isochrones relative to another set of initial points such as the set of vertically aligned points at a possible core site (located at $x=C$ ). Such isochrones would show where the ice currently in the core was located at earlier times. A set of core-relative isochrones, which we call pre-cores, is shown in Figure 5 for a core at $0.2 L$.

Figure 6 illustrates why pre-cores are relevant to the problem of turning open folds into recumbent folds. If a disturbance originates at point $(\mathbf{p})$ with a leading-limb angle equal to the pre-core slope angle ( $24^{\circ}$ in this example), this limb will be near-vertical when the disturbance reaches point $(\mathbf{q})$ at $x=0.2 L$. This disturbance would appear as an obvious fold-in-progress in a core sample taken at this point (provided that it is small enough to fit in the core cross-section). Further downstream at point (r) it will appear as a nearly horizontal $\left(\theta=176^{\circ}\right)$ segment.

A graphical way to use these pre-cores would be to overlay them with plots of representative disturbances. Any portion of a disturbance that is steeper than the corresponding pre-core will be overturned at the core location. Portions that are not as steep as the pre-core will not be overturned in this core, although overturning further downstream is still possible. Pre-cores can also be generated for more complicated flow models, as long as particle paths and stratigraphic isochrones can be calculated.

Figure 7 a shows contours of the angles of the pre-cores in Figure 5, with the same particle paths shown. We denote this angle field as $\theta_{\mathrm{f}}(x, z ; C)$. It is a function of the point in the ice sheet, and of the core location. Figure $7 \mathrm{~b}$ shows the angles along three particle paths for segments that have an angle of $90^{\circ}$ at $x=C$. The rotation through vertical is abrupt for deep paths, and much more gradual for paths near the surface. This contrast in rotation rates is largely a result of the larger vorticity number at depth, though the lower velocity near the bed makes the contrast stronger when plotted against distance $(x)$ rather than against time.

In Figure $7 b$, the angle at point $(\mathbf{p})$ is close to the minimum along its particle path. This means that a segment with a slope angle of $24^{\circ}$ is not rotating at this point in the flowband. In Figure 7a, the same property is seen in the fact that the angle contour is parallel to the particle path at this point. At this point, $\theta_{\mathrm{r}} \approx 24^{\circ}$.

Consider a segment with an angle of $60^{\circ}$ near the surface on the same particle path (the middle diamond). As it moves downstream, it is flattened (angle decreasing) until it reaches point $(\mathbf{p})$. At this point it starts rotating in the other direction. It passes through vertical at (q) and continues rotating toward horizontal in the downstream direction. Disturbances can be flattened when $W_{\mathrm{k}}$ is small (dominant pure shear), but farther along the path, $W_{\mathrm{k}}$ grows to the point that the flattening changes to steepening and overturning.

The pre-core slope angles $\theta_{\mathrm{f}}(x, z ; C)$ contoured in Figure $7 \mathrm{a}$ are the angles of segments that will reach vertical $(\theta$ $\left.=90^{\circ}\right)$ in the core at $x=\mathrm{C}$. At any point in the ice sheet, we can also think of $\theta_{\mathrm{f}}$ as a threshold for disturbances that will be overturned in an ice core at $C$. Segments that are steeper than $\theta_{\mathrm{f}}$ will overturn prior to reaching the core location, $C$. Segments with a smaller $\theta<\theta_{\mathrm{f}}$ will not overturn before they get to $C$ and might never do so.

The f subscript in $\theta_{\mathrm{f}}$ alludes to the finite time interval over which this rotation to vertical occurs. This is in contrast to the critical wrinkle angle, $\theta_{\mathrm{r}}$, derived by Waddington and others (2001), which focuses on the rate at which a segment is rotating at a point. $\theta_{\mathrm{f}}$ is a function of both the chosen core

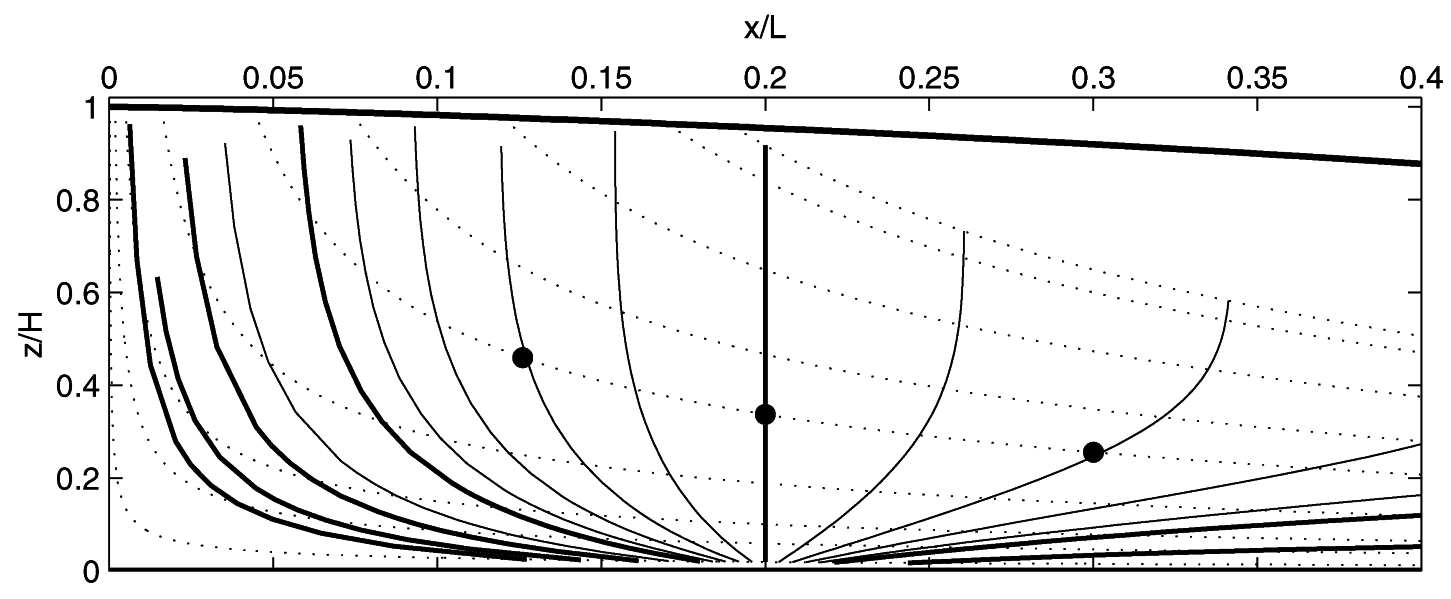

Fig. 5. Core-relative isochrones (pre-cores) for an ice sheet with an $H$ to $L$ ratio of 1:50 and a core at $0.2 L(10 H)$. The heavier lines are at $1 T$ intervals. Selected particle paths are drawn as dotted lines. 


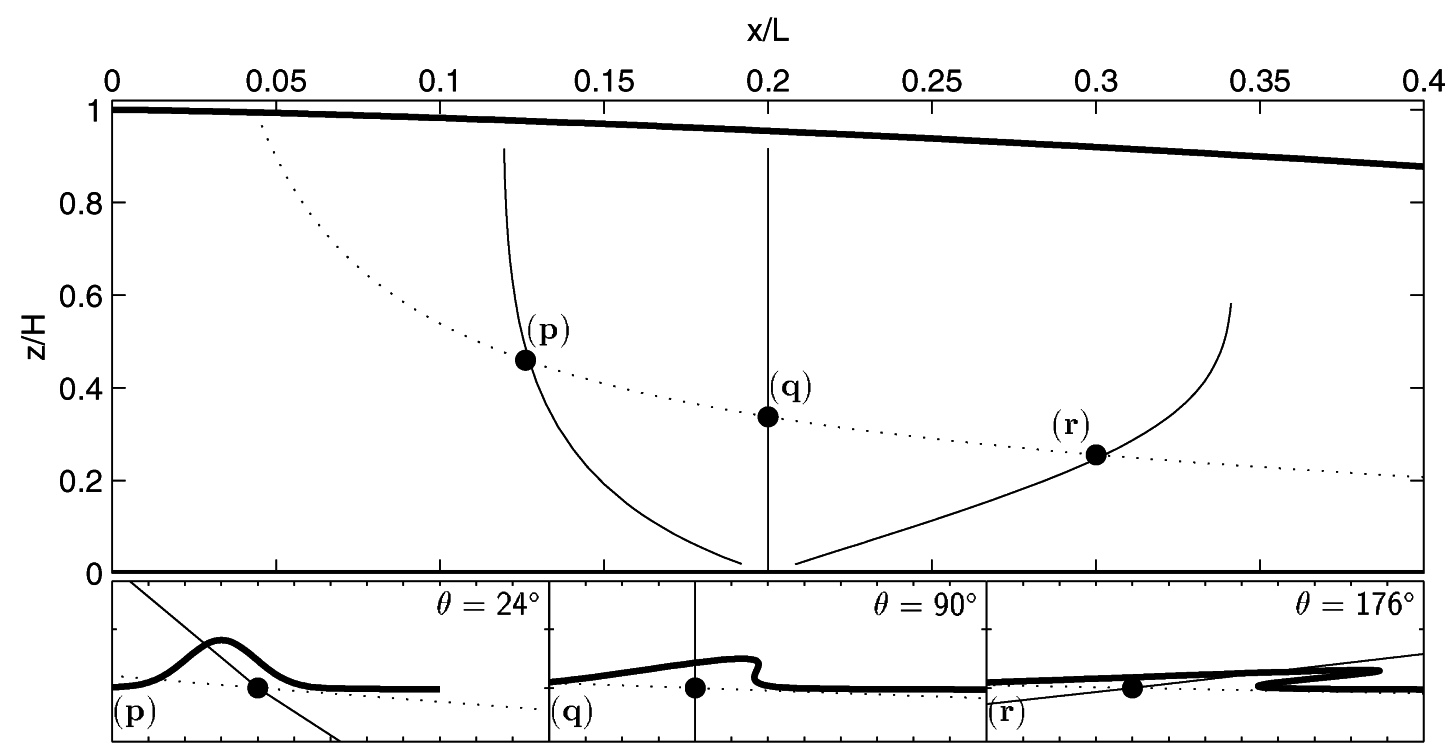

Fig. 6. The relation of pre-cores to the folding of a sample disturbance. A particle path (dotted line) is shown with three pre-cores (solid line). The pre-cores are at $0.4 T$ before and after the core at $0.2 \mathrm{~L}(10 \mathrm{H})$. The small figures underneath show a representative disturbance at these three points, $(\mathbf{p}),(\mathbf{q})$ and $(\mathbf{r})$. These three plots have the same scale, but a different aspect ratio from the larger plot. The particle path and pre-core through the center point is included on each subplot. The number in the upper right corner is the angle of the pre-core at that point.

location, $C$, and the current location of the ice segment, while $\theta_{\mathrm{r}}$ is a function of only the velocity gradient at the current location. As noted above, in connection with Figure 7, along a particle path $\theta_{\mathrm{f}}$ decreases, reaches a minimum and then increases. At its minimum, $\theta_{\mathrm{f}}$ equals the local $\theta_{\mathrm{r}}$. For points upstream, the $\theta_{\mathrm{f}}$ corresponding to this path (and core) is smaller than the local $\theta_{\mathrm{r}}$. For points downstream from the minimum, the opposite is true.

The finite perpendicular material line (FPM) of Grasemann and Vannay (1999) is similar to our pre-cores. They show that currently inverted metamorphic zones in rock could have originated with tilted but otherwise normal (cold over hot) metamorphic isotherms. The FPM is the pre-deformation alignment of a set of rocks sampled in the currently deformed terrane.

\section{DEFORMATION-GRADIENT TENSOR}

Pre-cores and their slope angle, $\theta_{\mathrm{f}}$, give an idea of how ice segments rotate as they move along particle paths toward the ice-core location. In particular they show which disturbances will be vertical in a core sample. We are also interested in layer disturbances that may not be exactly vertical in a core. A more general tool for relating segments, or small structures in general, at one point in the ice-flow field to their strained form at a core sample is the deformation-gradient (or finite-strain) tensor. In this section we explain how this tensor is calculated with our flow model, and use it to calculate $\theta_{\mathrm{f}}$.

\subsection{The strain from reference point to current point}

The deformation-gradient tensor is a measure of the strain that occurs over a finite distance in a flow field. Let $\mathrm{d} \mathbf{X}$ (with components $\mathrm{d} X$ and $\mathrm{d} Z$ ) be a small segment at a reference point such as $(\mathbf{p})$ in Figure 6. The corresponding de- formed segment, $\mathrm{d} \mathbf{x}$ at the current point (e.g.(q)), can be expressed as a linear function of $\mathrm{d} \mathbf{X}$.

$$
\mathrm{d} \mathbf{x}=\mathbf{F}_{\langle(\mathbf{p})(\mathbf{q})\rangle} \cdot \mathrm{d} \mathbf{X},
$$

where $\mathbf{F}_{\langle(\mathbf{p})(\mathbf{q})\rangle}$ is a tensor mapping $\mathrm{d} \mathbf{X}$ onto $\mathrm{d} \mathbf{x}$. The bracketed subscripts denote the reference and current points. When it is clear which points we are referring to, we will just write $\mathbf{F}$. The component $F_{x x}=\partial x / \partial X$ maps a reference horizontal segment (or the horizontal component of a segment), $\mathrm{d} X$, onto the current horizontal component, $\mathrm{d} x$. $F_{x z}$ maps $\mathrm{d} Z$ onto $\mathrm{d} x$ (vertical to horizontal). In terms of the tensor components, Equation (18) is

$$
\left[\begin{array}{c}
\mathrm{d} x \\
\mathrm{~d} z
\end{array}\right]=\left[\begin{array}{lll}
F_{x x} \mathrm{~d} X & + & F_{x z} \mathrm{~d} Z \\
F_{z x} \mathrm{~d} X & + & F_{z z} \mathrm{~d} Z
\end{array}\right] .
$$

The tensors for the deformations shown in Figure 1 are

$$
\text { (a) } \mathbf{F}=\left[\begin{array}{cc}
3 / 2 & 0 \\
0 & 2 / 3
\end{array}\right] \text { and (b) } \mathbf{F}=\left[\begin{array}{ll}
1 & 1 \\
0 & 1
\end{array}\right] \text {. }
$$

We are particularly interested in the rotation of layer segments. If $\Theta$ is the angle of the segment at the reference point, then the current angle, $\theta$, satisfies

$$
\tan (\theta)=\frac{\mathrm{d} z}{-\mathrm{d} x}=\frac{-F_{z x}+F_{z z} \tan (\Theta)}{F_{x x}-F_{x z} \tan (\Theta)} .
$$

(The negative signs result from defining angles relative to the $-x$ coordinate.)

F can be calculated from closely spaced particle paths, much as finite strain is calculated in the field or laboratory; however, we can also find it using a set of differential equations

$$
\dot{\mathbf{F}}=\mathbf{L} \cdot \mathbf{F} \text {. }
$$

$\mathbf{L}$ (Equation (3)) is the spatial gradient of the velocity relative to the current-point coordinate frame, while $\dot{\mathbf{F}}$ is the gradient of the velocity relative to the reference-point frame. 


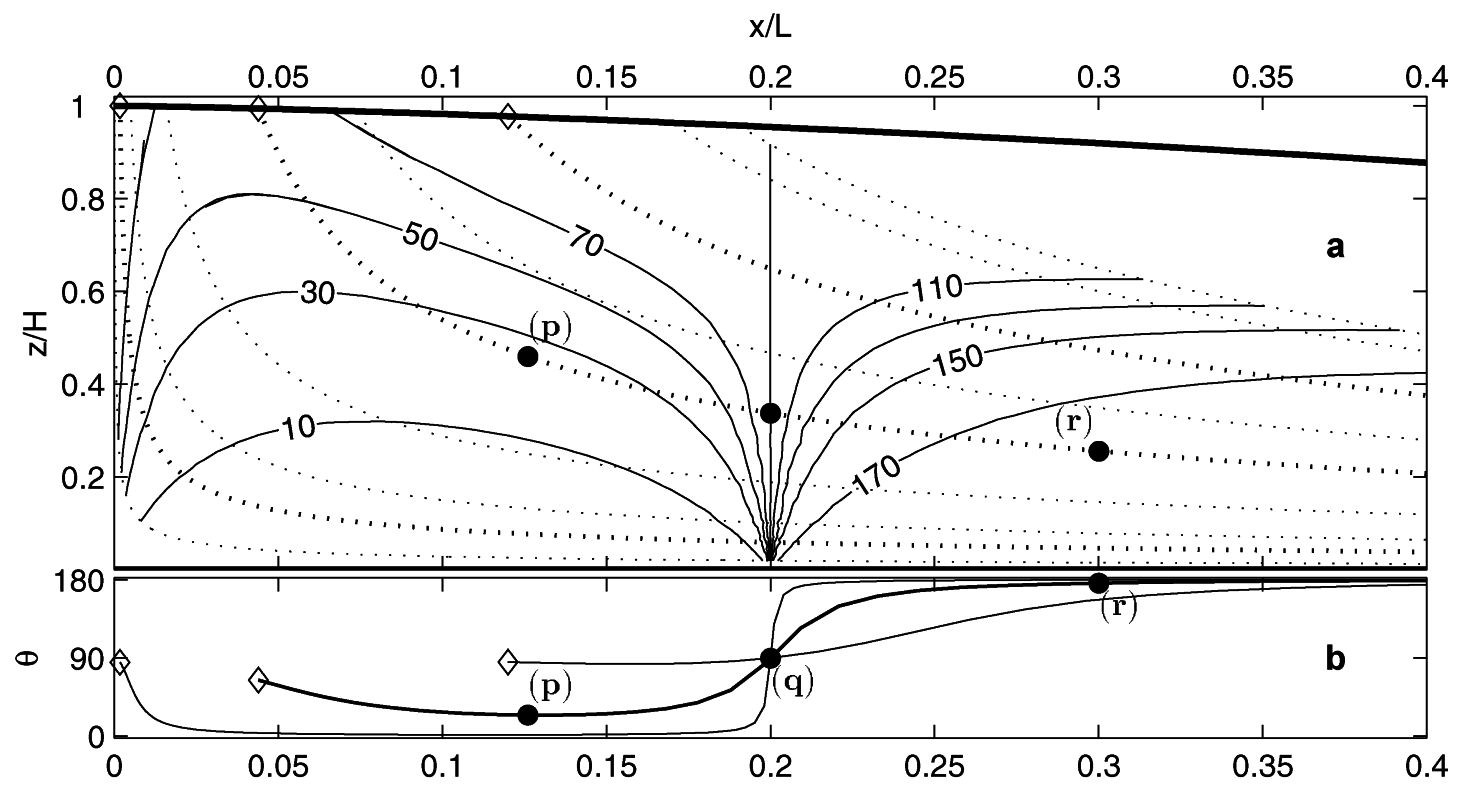

Fig. 7. (a) Contours of the pre-core angles, $\theta_{\mathrm{f}}$, in degrees. (b) Pre-core angles along selected particle paths (marked with $\diamond$ at the surface). (p), (q) and (r) points in Figure 6 are also shown.

We can get a rough idea of where Equation (22) comes from by writing

$$
\frac{\mathrm{d}}{\mathrm{d} t}\left(\frac{\partial x}{\partial X}\right)=\frac{\partial}{\partial X} \frac{\mathrm{d} x}{\mathrm{~d} t}=\frac{\partial v}{\partial X}=\frac{\partial v}{\partial x} \frac{\partial x}{\partial X} .
$$

See Malvern (1969, section 4.5) for details.

We solve Equation (22) for $\mathbf{F}$ when we calculate a particle path (Equation (17)), using $\mathbf{I}$, the identity matrix, as its initial value. These form a differential-equation system of six variables $(x, z$ and the four strain terms). $\mathbf{L}$ is calculated with an eight-point finite difference to maximize continuity. The error tolerances have to take into account the widely differing magnitudes of the variables. The result is a series of $\mathbf{F}$ tensors, one for each point along the particle path, relating $\mathrm{d} \mathbf{x}$ at that point to $\mathrm{d} \mathbf{X}$ at the path start. The components of such a series of tensors are shown in Figure $8 \mathrm{~b}-\mathrm{d}$. The particle path is shown in Figure $8 \mathrm{a}$. In Figure $8 \mathrm{~b}$ and $\mathrm{c}$ the reference point is at the surface of the ice sheet. The diagonal components of the tensor, $F_{x x}$ and $F_{z z}$, start at 1 , while the two shear components, $F_{z x}$ and $F_{x z}$, start at 0. $F_{z x}$ remains too close to zero to plot with the other components (it is slightly negative, with some increase in magnitude near the terminus).

The determinant of $\mathbf{F}$ is the Jacobian (Malvern, 1969, section (equation (4.5.25)) relating an infinitesimal deformed volume at the current point to the undeformed volume at the reference point. Since ice is incompressible, this determinant must be equal to unity at all points along the particle path.

$$
1=|\mathbf{F}|=F_{x x} F_{z z}-F_{z x} F_{x z} .
$$

While $F_{z x} F_{x z}$ is small, $F_{z z} \approx 1 / F_{x x}$. When $x / L<1 / 3, F_{x x}$ is approximately linear in $x$. (See Appendix B for details.) This also means that $F_{z z} \approx x_{0} / x$, so that the amplitude of a disturbance decreases inversely with the distance traveled away from the divide. A disturbance observed at $10 H$ would be approximately half as high as it had been at $5 H$, regardless of the observation depth or slope.

\subsection{Choosing the core as reference point}

The reference point of $\mathbf{F}$ can be changed. Taking the inverse, $\mathbf{F}_{\langle(\mathbf{p})(\mathbf{q})\rangle}^{-\mathbf{1}}$, interchanges the reference and current points, $(\mathbf{p})$ and $(\mathbf{q})$, in effect undoing the strain.

$$
\mathrm{d} \mathbf{X}=\mathbf{F}_{\langle(\mathbf{p})(\mathbf{q})\rangle}^{-1} \cdot \mathrm{d} \mathbf{x}=\mathbf{F}_{\langle(\mathbf{q})(\mathbf{p})\rangle} \cdot \mathrm{d} \mathbf{x}
$$

We use such an inverse to shift the reference point from the surface, $(\mathbf{s})$, to the core, $(\mathbf{q})$.

$$
\mathbf{F}_{\langle(\mathbf{q})(\mathbf{p})\rangle}=\mathbf{F}_{\langle(\mathbf{s})(\mathbf{p})\rangle} \cdot \mathbf{F}_{\langle(\mathbf{s})(\mathbf{q})\rangle}^{-1}
$$

The components for such a shifted set of $\mathbf{F}$ tensors are shown in Figure 8d and e.

Since a segment aligned with the core at (q) (Fig. 6) is vertical $\left(\Theta=90^{\circ}\right)$, its angle at other points on the particle path can be calculated from a modified version of Equation (21),

$$
\tan \theta_{\mathrm{f}}=\frac{-F_{z x} 0+F_{z z} \mathrm{~d} Z}{F_{x x} 0-F_{x z} \mathrm{~d} Z}=\frac{F_{z x}}{-F_{x z}} .
$$

This is the pre-core slope angle, $\theta_{\mathrm{f}}$, contoured in Figure 7 and plotted in Figure 8f.

The minimum point of $\theta_{\mathrm{f}}$ along the path, where $\theta_{\mathrm{f}}=\theta_{\mathrm{r}}$, is a consequence of the variation of the velocity gradient, $\mathbf{L}$, along the path. As $\mathbf{L}$ changes, $\theta_{\mathrm{r}}$ decreases, leading to the change in rotation direction for certain segments (section 1.2). On the other hand, the rapid change in $\theta_{\mathrm{f}}$ while it passes through $90^{\circ}$ (at the core) does not depend on $\mathbf{L}$ changing. This rapid rotation through vertical occurs even when $\mathbf{L}$ is assumed to be constant (see Appendix G).

\subsection{Patterns of segment deformation}

The $\theta_{\mathrm{f}}$ plot in Figure 8 (and Fig. 7b) shows the angle evolution of an ice segment that is vertical at the core. The series of $\mathbf{F}$ tensors along a particle path can be used to track the history of any segment. Figure 9 shows the angle evolution that a set of ice-layer segments would undergo as they travel along a particle path. The history varies depending on the initial angle $\Theta$ of the segment. On the left, pure shear dominates, because the path is near the surface. All segments rotate toward horizontal $\left(0^{\circ}\right.$ and $\left.180^{\circ}\right)$. Segments near vertical 
$\mathrm{x} / \mathrm{L}$
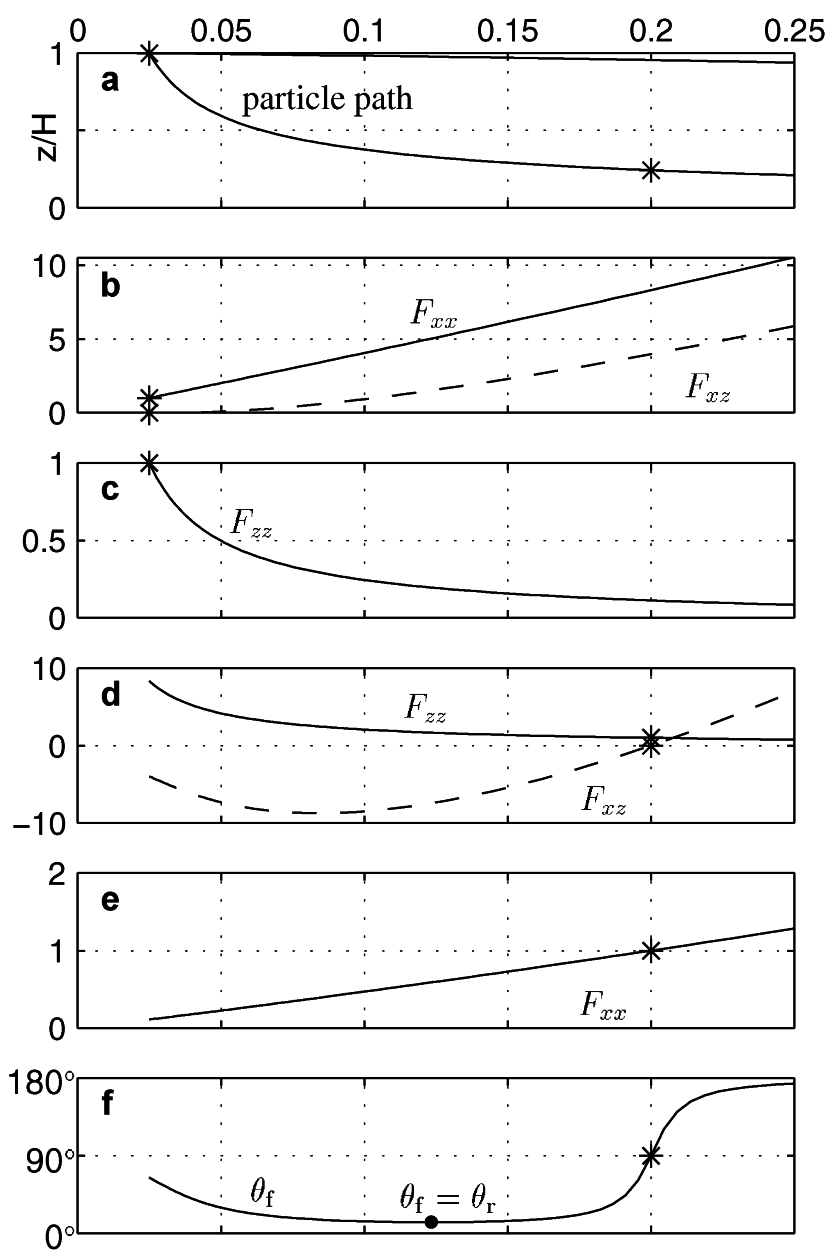

Fig. 8. $\mathbf{F}$ components of (dimensionless) finite strain along a particle path ( $a)$. In $(b)$ and $(c)$ the reference point $(*)$ is at the surface; in $(d)$ and $(e)$ it is at $x=0.2 L$. (f) Finitestrain threshold, $\theta_{\mathrm{f}}=\tan ^{-1}\left(-F_{z z} / F_{x z}\right)$.

are under compression (the shaded region) while near-horizontal segments are under extension. As the proportion of simple shear increases, the range of angles under compression shifts to angles less than $90^{\circ}$. All segments with an angle less than that of the particle path itself, $\theta_{\mathrm{p}}$, continue to rotate toward $0^{\circ}$.

\section{PREDICTING THE ORIGIN SITE OF OBSERVED FOLDS}

We can start with an observed structure in a core, and attempt to predict where it originated and what its shape was there. The amount of information that we can deduce about the original disturbance depends on how much information we can glean from the deformed structure. To further constrain the point of origin, we may have to make some additional assumptions about the source disturbance. For example, if we assume that gentle wrinkles are more likely to occur than steeper ones, then the point where the $\theta_{\mathrm{f}}$ curve has a minimum may be the most likely point of origin of a disturbance.

\subsection{Minimum disturbance-angle assumption}

In cores such as GISP2, stratigraphic layers with tilts of 5$20^{\circ}$ have been observed (Alley and others, 1995). With our deformation model, we can project these tilted layers upstream and downstream from the observed location at $9 \mathrm{H}$
$\mathrm{X} / \mathrm{L}$

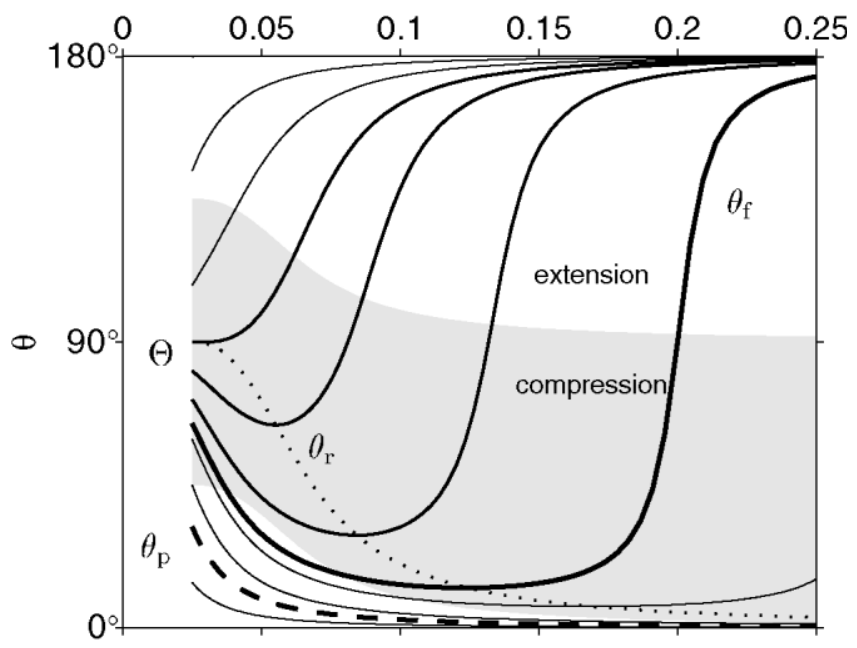

Fig. 9. Rotation of segments of various initial slope angle $\Theta$ along a particle path (Fig. 8a). The line marked $\theta_{\mathrm{f}}$ is the pre-core angle for a core at $0.2 L$. The heavy dashed line is the angle of the particle path, $\theta_{\mathrm{p}}$. Shading marks where segments are undergoing shortening. The dotted line marks the zerorotation angle, $\theta_{\mathrm{r}}$, which was used to formulate a folding criterion in earlier studies (Waddington and others, 2001).

from the divide $(\approx 0.1 L)$. Figure 10 a shows the angle history of the leading limb of a recumbent fold at a depth of $0.8 \mathrm{H}=$ $2400 \mathrm{~m}$ in the core. At the core site, its angle is slightly past vertical $\left(100^{\circ}\right)$. This recumbent fold could have originated from a disturbance with leading-limb angle of about $10^{\circ}$ at $x \approx 6 H$. This disturbance has been largely unchanged or flattening for most of its history, with most of its rotation occurring close to the core site. Figure $10 \mathrm{~b}$ is similar but for a $20^{\circ}$ segment at $0.88 H=2640 \mathrm{~m}$ depth. Because the azimuthal orientation of the core is unknown, we show the history for segments that tilt $20^{\circ}$ in both the upstream and downstream directions. During their prior history, the minimum angles are practically the same for both segments (about $5^{\circ}$ ). The most likely origin point of this disturbance is a broad region around $5 H$. For similar segments at a depth of $0.92 \mathrm{H}$, the minimum angle is closer to $3^{\circ}$.

It is apparent from Figure 7 that the $\theta_{\mathrm{f}}$ minimum is further upstream the deeper we look in the core (in Figure $7 \mathrm{a}$ the minimum $\theta_{\mathrm{f}}$ occurs along a particle path where the $\theta_{\mathrm{f}}$ contour is tangent to the path). However, as is evident from the curves in Figure $7 \mathrm{~b}$, the deeper we look in the core, the less ability we have to resolve that disturbance injection point, because on the deeper paths $\theta_{\mathrm{f}}$ has much broader minima. Thus disturbances with similar small angles that fall anywhere in a broad region can all cause overturning folds at the core site downstream.

We emphasize the fact that, when layer segments overturn, they overturn very quickly. The characteristic timescale $T=H / \dot{b}$ for strain in central Greenland is 10000 years (Table 1). The steady-state age of ice at $0.8 \mathrm{H}$ depth at the location of the GISP2 core is 25000 years. The time for a segment to evolve from its upstream minimum value to $90^{\circ}$ (vertical) at the core is about 700 years. In Figure $10 \mathrm{~b}$, at a deeper level, the age is 40000 years, and the time from minimum slope to overturning is about 1000 years. In contrast, the time taken to rotate from $20^{\circ}$ through vertical to $160^{\circ}$ is only about 200 years, i.e. rotation through angles differing significantly from horizontal happens very quickly. 

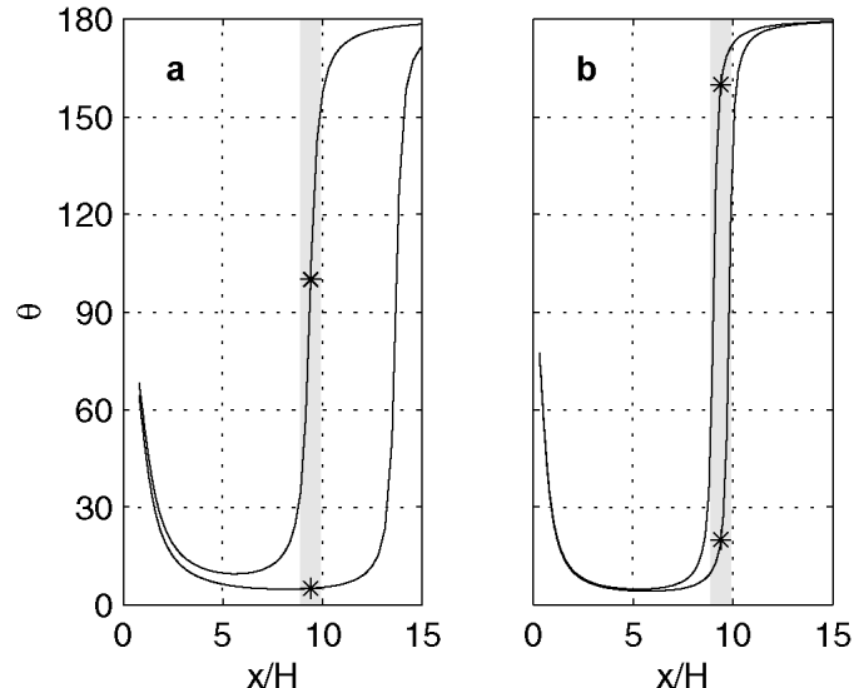

Fig. 10. Predicted histories of observed disturbances in a core at $9 H$ ( $\approx$ GISP2). (a) History of a $100^{\circ}$ slightly overturned core segment at a depth of $0.8 \mathrm{H}\left(10^{\circ}\right.$ minimum value). For comparison the history of a segment that is at $5^{\circ}$ in the core at the same depth is also plotted. (b) Histories of segments that are oriented at $20^{\circ}$ and $160^{\circ}$ at $0.88 \mathrm{H}$ depth in the core $\left(5^{\circ}\right.$ minimum values).

These steady-state depth-age values do not take into account the much lower accumulation rate during glacial times, so they are younger than measured core ages. However, the overturn times fall well within the current interglacial period, so the deformation causing this folding is not affected by the earlier accumulation rates.

Figure 11 shows a similar case with a different flowband model. The bed approximates the radar profile from the Greenland summit (Jacobel and Hodge, 1995; Castelnau and others 1998). The velocity profile $\hat{u}(x, \hat{d})$ has been adapted from a finite-element flowband model (Bolzan and others, 1995; Nereson and others, 1998). Most of the difference between Figures 10 and 11, once the bed slope is accounted for, can be attributed to the polythermal nature of the finite-element model.

It should be noted that with bed undulations (Fig. 11), the $\theta_{\mathrm{f}}$ curve has multiple local minima, suggesting that a fold observed in a core could have originated as a shallow disturbance in any of several distinct localities. If the segment angles are measured relative to the local steady-state isochrone (Waddington and others, 2001) this oscillation in $\theta_{\mathrm{f}}$ is reduced, but not eliminated (Jacobson, 2001). These minima of $\theta_{\mathrm{f}}$ are also points where the rotation rate is zero. Thus the presence of bed undulations increases the need to look at the finite strain along a particle path, and not just the local rotation rate (Waddington and others, 2001), when evaluating fold potential.

\subsection{Symmetric disturbance assumption}

We could also constrain the disturbance location if we knew its initial shape. If the disturbance is the result of layers draping over a transient "hard lump" (Fig. 3) it might be reasonable to assume that, before shearing, the disturbance was symmetrical as depicted at point $(\mathbf{p})$ in Figure 6. If we observe the fold at point (q) (in a core), and measure the angles of the leading limb and trailing limb, we can constrain the location of point $(\mathbf{p})$, by running the wrinkle
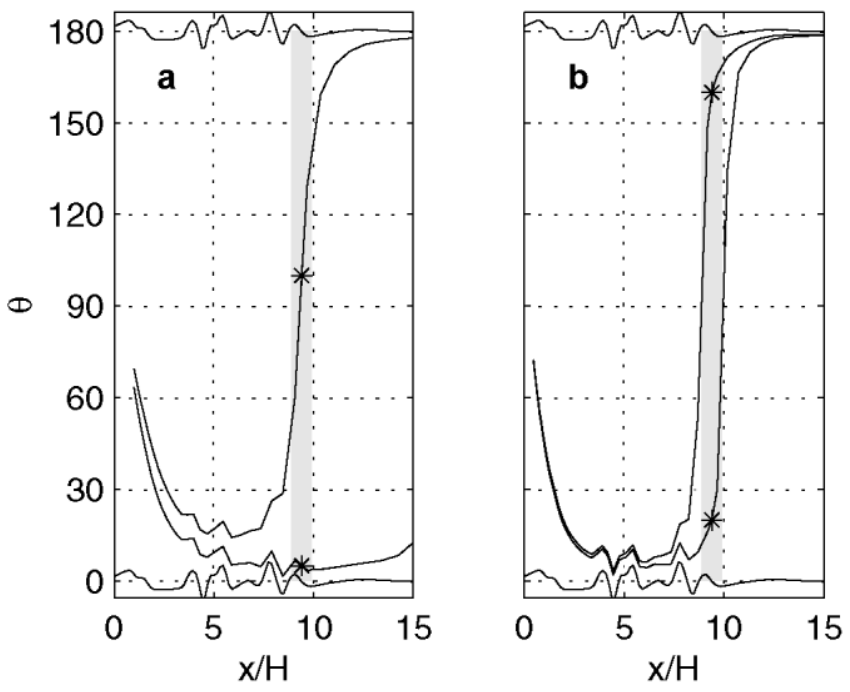

Fig. 11. Predicted histories of observed disturbances in a core at $9 H(\approx$ GISP2), as in Figure 10, but with bed and velocity profiles that better approximate the Greenland summit area. (a) History of a $100^{\circ}$ slightly overturned core segment at a depth of $0.8 \mathrm{H}\left(15^{\circ}\right.$ minimum slope upstream $)$. (b) Histories of segments that are oriented at $20^{\circ}$ and $160^{\circ}$ at $0.88 \mathrm{H}$ depth in the core $\left(2-4^{\circ}\right.$ minimum slope upstream). Relative to the bed slope these minima are (a) $15^{\circ}$ and (b) 5-6. The bed-slope angle is plotted at the top and bottom (centered on $180^{\circ}$ and $0^{\circ}$ ).

backwards from the core until its shape becomes symmetrical. See Appendix D for details of such a calculation.

Thorsteinsson and Waddington (2002) investigated the deformation pattern in an anisotropic layer in which the $c$ axes clustered in a cone, and the cone orientation varied with position; non-symmetric wrinkles were created in pure shear, which approximates the stress pattern in the upper part of an ice sheet near a divide. However, if the fabric was everywhere vertical, and varied spatially only in its degree of clustering about the vertical (cone angle), then we could expect that symmetric wrinkles would be created in pure shear. If a search for asymmetric origin of an observed wrinkle finds no solution (as shown in Appendix D), this could be an indication of variable and non-vertical fabrics at the source region. In such a case we would have to use a flow model that takes the fabrics into account.

\section{DISGUSSION}

While the probability of a core containing a fold increases downstream, and deeper into the ice sheet, this fold may be harder to recognize. The interval during which a fold is obviously overturning is short, because the overturning limb rotates rapidly. Once a segment has overturned, it continues to rotate toward the orientation of the undisturbed isochrones. Since ice lacks reliable "up" indicators (Alley and others, 1995), the fold limbs will merge back into the otherwise undisturbed stratigraphy, leaving a stratigraphic "error" that may be undetectable in an ice core.

Folds can also be difficult to identify in radar layering. First, as noted above, folds can overturn very quickly when the leading limb is noticeably non-horizontal; the leading limb spends very little time at a high angle to the normal stratigraphy. When a fold is sub-parallel to the undisturbed stratigraphy, it is unlikely to be identified as a fold by radar. 
Second, as suggested by Waddington and others (2001), folds may appear on an increasing hierarchy of spatial scales. The youngest disturbances seen in the GISP2 core occur on scales of centimeters. At scales of decimeters to meters, icecore records can be noticeably compromised. Folds of such small scale cannot be detected by ice-penetrating radar; in ice, the wavelength of the energy pulse is many meters to decameters, and the separation between successive radar traces is typically many meters. At $2-3 \mathrm{~km}$ below the surface, a fold of this small scale will also be a very weak point-diffractor. At spatial scales of tens to hundreds of meters, folds could be imaged by typical ice-penetrating radar if the limbs have not merged back with the undisturbed stratigraphy. Folds on these large scales are probably relatively rare. Finally, it has been suggested (Robin, 1983) that radar may detect stratigraphic disturbances through the existence of an "echo-free zone" deep in the ice sheet, i.e. through the inability of radar to generate coherent reflections from layers that have been geometrically disturbed by folding or other heterogeneous flow. On the other hand, absence of detectable radar layers does not always indicate disturbed stratigraphy; it can also result from power and sensitivity limitations of radar echo-sounding equipment, i.e.the connection between the lack of echo and the disturbance is not conclusive.

Folding has been found in the lower $20 \%$ of ice in the central Greenland ice cores (Alley and others 1995); however, ice cores are very poor at revealing structures larger than a few decimeters. Ice-penetrating radar also hints at folding in the deep echo-free zone (Robin, 1983); however, as noted above, radar may be poor at detecting structures smaller than tens to hundreds of meters. Direct evidence for folding at intermediate scales is even weaker, due to our inability to make critical observations. Nevertheless, the limited data suggest that folds are more common at depth, and our models also predict this. However, it is important to note that our models also show that folds may be encountered virtually anywhere in an ice sheet, except close to the surface and under a stable ice divide.

Although our examples assume steady flow and a frozen flat bed, our approach is equally applicable to transient ice sheets on rough beds, and to ice sheets with wet beds and sliding basal ice. We did not include basal sliding here, primarily to keep our ice-flow model simple; however, wherever an ice sheet has a component of ice flow due to internal shear deformation, folds such as we have described are possible. Even where internal shear deformation is insignificant, such as in ice streams, in ice shelves and over large subglacial lakes, the ice can contain folds that were acquired in shearing flow upstream.

In addition, transitions between sliding and frozen sections of partially frozen beds introduce local strain variations in the flow, and have the potential of altering stratigraphy. If these boundaries are transient, then their migration is likely to position some layer sections in orientations where they can subsequently be overturned by passive steady flow.

\section{GONGLUSIONS}

The kinematics of large-scale ice-sheet flow can deform gentle open folds into order-disturbing recumbent folds. To extend the results of Waddington and others (2001) to ac- count for spatially variable strain rates, we have used three concepts: $\theta_{\mathrm{r}}$ the no-rotation angle, $\theta_{\mathrm{f}}$ the finite-strain threshold angle, and pre-cores.

From the velocity gradient at a point, we calculate $W_{\mathrm{k}}$, the kinematic vorticity number which measures the relative mix of pure and simple shear, and $\theta_{\mathrm{r}}$, the angle that is not rotating at that point. Segments steeper than $\theta_{\mathrm{r}}$ are rotating toward vertical and overturning, while gentler ones are being flattened. But because segments move along paths into regions of higher $W_{\mathrm{k}}$, segments that were not rotating will begin to overturn. So, while the $\theta_{\mathrm{r}}$ criterion of Waddington and others (2001) is an approximate indicator of stability against recumbent folding, it is still optimistic about downstream stratigraphic integrity. Our finite-strain analysis can quantify this.

By calculating the finite-strain deformation-gradient tensor, $\mathbf{F}$, along a particle path, we see how a disturbance segment rotates over a finite time interval. Given our limited understanding of the dynamic processes that inject disturbances, the most useful form of the angle-rotation function specifies a vertical segment at a reference (observation) point, and calculates the corresponding angle at other points along the path. We have called this the finitestrain threshold angle, $\theta_{\mathrm{f}}$. At any point, this is the angle of the segment that will be in the process of overturning when it reaches the reference point.

$\theta_{\mathrm{r}}$ is a minimum angle on the $\theta_{\mathrm{f}}$ curve, i.e. the gentlest segment that will overturn at the reference point. A segment can flatten for a period of time, then reach the local $\theta_{\mathrm{r}}$ and proceed to steepen and overturn. Since this minimum can be broad, it gives little indication of how soon the overturn will occur. For that, the full $\theta_{\mathrm{f}}$ calculation in this paper is needed.

Pre-cores, or core-relative isochrones, extend the concept of the $\theta_{\mathrm{f}}$ curve to multiple particle paths. The reference isochrone is defined along a hypothetical ice core, and upstream pre-cores give the location of the core ice at earlier times. They also give the slope of segments that will be vertical in the core. A $\theta_{\mathrm{f}}$ curve gives the pre-core slope angle along its path. Thus the pre-cores and the $\theta_{\mathrm{f}}$ contours are graphical ways of showing just how steep disturbances must be upstream in order to be overturned in the core.

Other processes, such as shear-band development or various three-dimensional flow effects, can also disrupt stratigraphy. In that sense, our calculations may still err on the optimistic side when assessing stratigraphic integrity. Our analysis identifies scale parameters for processes that could generate initial disturbances. A process that cannot generate an adequate disturbance can be ruled out as a core-disrupting process.

\section{ACKNOWLEDGEMENTS}

This research was supported by U.S. National Science Foundation grants OPP-9123660, OPP-9420648 and OPP9726113. We are grateful to G. Raymond, R. Fletcher, B. Hallet and T. Thorsteinsson for valuable insights. N. F. Glasser (Scientific Editor) and M.J. Siegert provided helpful reviews of the manuscript.

\section{REFERENGES}

Alley, R. B., A.J. Gow, S. J. Johnsen, J. Kipfstuhl, D. A. Meese and Th. Thorsteinsson. 1995. Comparison of deep ice cores. Nature, 373(6513), 393-394. 
Alley, R. B., A. J. Gow, D. A. Meese, J. J. Fitzpatrick, E. D. Waddington and J. F. Bolzan. 1997. Grain-scale processes, folding and stratigraphic disturbance in the GISP2 ice core. 7. Geophys. Res., 102(C12), 26,81926,830 .

Anandakrishnan, S., R. B. Alley and E. D. Waddington. 1994. Sensitivity of the ice-divide position in Greenland to climate change. Geophys. Res. Lett., 21 (6), 441-444.

Azuma, N. and K. Goto-Azuma. 1996. An anisotropic flow law for ice-sheet ice and its implications. Ann. Glaciol., 23, 202-208.

Bobyarchick, A. R. 1986. The eigenvalues of steady flow in Mohr space. Tectonophysics, 122(1-2), 35-51.

Bolzan, J. F., E. D. Waddington, R. B. Alley and D. A. Meese. 1995. Constraints on Holocene ice-thickness changes in central Greenland from the GISP2 ice-core data. Ann. Glaciol., 21, 33-39.

Castelnau, O. and 7 others. 1998. Anisotropic behavior of GRIP ices and flow in central Greenland. Earth Planet. Sci. Lett., 154(1-4), 307-322.

Conway, H., B. L. Hall, G. H. Denton, A. M. Gades and E. D. Waddington. 1999. Past and future grounding-line retreat of the West Antarctic ice sheet. Science, 286, 280-283.

Dahl-Jensen, D., T. Thorsteinsson, R. Alley and H. Shoji. 1997. Flow properties of the ice from the Greenland Ice Core Project ice core: the reason for folds? 7. Geophys. Res., 102(C12), 26,831-26,840.

Fuchs, A. and M. C. Leuenberger. 1996. $\delta^{18} \mathrm{O}$ of atmospheric oxygen measured on the GRIP ice core document stratigraphic disturbances in the lowest 10\% of the core. Geophys. Res. Lett., 23(9), 1049-1052.

Grasemann, B. and J. C. Vannay. 1999. Flow controlled inverted metamorphism in shear zones. 7. Struct. Geol., 21 (7), 743-750.

GRIP Project Members. 1993. Climate instability during the last interglacial period recorded in the GRIP ice-core. Nature, 364(6434), 203-207.

Grootes, P. M., M. Stuiver, J.W. C. White, S. Johnsen and J. Jouzel. 1993. Comparison of oxygen isotope records from the GISP2 and GRIP Greenland ice cores. Nature, 366(6455), 552-554.

Hudleston, P. J. 1977. Similar folds, recumbent folds, and gravity tectonics in ice and rocks. 7. Geol., 85(1), 113-122.

Hudleston, P. J. and R. LeB. Hooke. 1980. Cumulative deformation in the Barnes Ice Cap and implications for the development of foliation. Tectonophysics, 66(1-3), 127-146.

Hutter, K. 1983. Theoretical glaciology; material science of ice and the mechanics of glaciers and ice sheets. Dordrecht, etc., D. Reidel Publishing Co./Tokyo, Terra Scientific Publishing Co.

Jacobel, R.W. and S.M. Hodge. 1995. Radar internal layers from the Greenland summit. Geophys. Res. Lett., 22(5), 587-590.

Jacobson, H.P. 2001. Folding of stratigraphic layers in ice domes. (Ph.D. thesis, University of Washington.)

Jacobson, H. P. and E. D. Waddington. 1996. Finite strain and folding in ice sheets. Eos, 77(46), Fall Meeting Supplement, F196.

Malvern, L. E. 1969. Introduction to the mechanics of a continuous medium. Englewood Cliffs, NJ, Prentice-Hall. (Engineering of the Physical Sciences.)

Marshall, S. J. and K. M. Cuffey. 2000. Peregrinations of the Greenland ice sheet divide in the last glacial cycle: implications for central Greenland ice cores. Earth Planet. Sci. Lett., 179(1), 73-90.

Means, W. D., B. E. Hobbs, G. S. Lister and P. F. Williams. 1980. Vorticity and non-coaxiality in progressive deformations. 7. Struct. Geol., 2(3), 371-378.

Nereson, N. A., G. F. Raymond, E. D. Waddington and R. W. Jacobel. 1998. Migration of the Siple Dome ice divide, West Antarctica. 7. Glaciol., 44(148), 643-652.

Paterson, W. S. B. 1994. The physics of glaciers. Third edition. Oxford, etc., Elsevier.

Ramberg, H. 1975. Particle paths, displacement and progressive strain applicable to rocks. Tectonophysics, 28(1-2), 1-37.

Raymond, C. F. 1983. Deformation in the vicinity of ice divides. F. Glaciol., 29(103), 357-373.

Reeh, N. 1988. A flow-line model for calculating the surface profile and the velocity, strain-rate, and stress fields in an ice sheet. 7. Glaciol., 34(116), 46-54.

Robin, G. de O. 1983. Radio-echo studies of internal layering of polar ice sheets. In Robin, G. de Q., ed. The climatic record in polar ice sheets. Cambridge, Cambridge University Press, 89-93.

Shampine, L. F. and M.W. Reichelt. 1997. The MATLAB ODE suite. SIAM 7. Scient. Comput., 18(1), 1-22.

Thorsteinsson, T. and E. D. Waddington. 2002. Folding in strongly anisotropic layers near ice-sheet centers. Ann. Glaciol., 35, 480-486.

Vaughan, D. G., H. F. J. Corr, C. S. M. Doake and E. D. Waddington. 1999. Distortion of isochronous layers in ice revealed by ground-penetrating radar. Nature, 398(6725), 323-326.

Vialov, S. S. 1958. Regularities of glacial shields movement and the theory of plastic viscours [sic] flow. International Association of Scientific Hydrology Publication 47 (Symposium at Chamonix 1958 - Physics of the Movement of the Ice), 266-275.
Waddington, E. D., J. F. Bolzan and R. B. Alley. 1995. The origin of folding in ice cores. [Abstract.] Eos, 76(17), Spring Meeting Supplement, S177.

Waddington, E. D., J. F. Bolzan and R. B. Alley. 2001. Potential for stratigraphic folding near ice-sheet centers. F. Glaciol., 47(159), 639-648.

\section{APPENDIX A}

\section{PRE-GORE SGALE DEPENDENGE}

Pre-cores are a simple tool for determining what disturbances could appear as overturned folds in an ice core. All that is needed is a flow model that can calculate particle paths and travel times along those paths. The paths are determined by the flowband geometry, which in our simple example is determined by its horizontal and vertical extent, $L$ and $H$. The core location $C$ determines the shape of the pre-cores. The time-scale $T$ for the ice sheet affects the contour interval of the pre-cores (as it does stratigraphic isochrones), but does not change their shape. We can gain further insight into pre-cores and the folding potential by looking at how the pre-core angles vary with these scaling factors.

Figure 12 shows how the pre-core angles vary with the core location $C$, while keeping $H / L$ constant. The solid set of lines with the core at $10 H$ are a subset of the contours in Figure 7. The dashed set are for a core at $20 H=0.4 L$. The gray lines mark where these angle contours are parallel to the particle paths, that is, where $\theta_{\mathrm{f}}=\theta_{\mathrm{r}}$.

For the inner portion of this simple ice sheet $(x \leq 0.4 L)$, the pre-core shapes are controlled primarily by the location of the core, specifically the $C / H$ ratio. The surface slope is small $\left(S^{\prime}(1-\hat{d}) \ll 0\right)$, and $w(x, z)$ is dominated by the $\dot{b} \hat{w}(\hat{d})$ term (Equation (9)). The distance to the terminus has limited direct impact on the velocity field, the particle paths and the isochrones. When the contours of $\theta_{\mathrm{f}}$ are plotted against $\hat{d}$ and $x$ (not shown), they show little variation as $H / L$ is varied, provided $C / H$ is held constant.

On the other hand, if we keep $C / L$ constant, the slope of a pre-core scales linearly with the $H / L$ ratio. If the length $L$

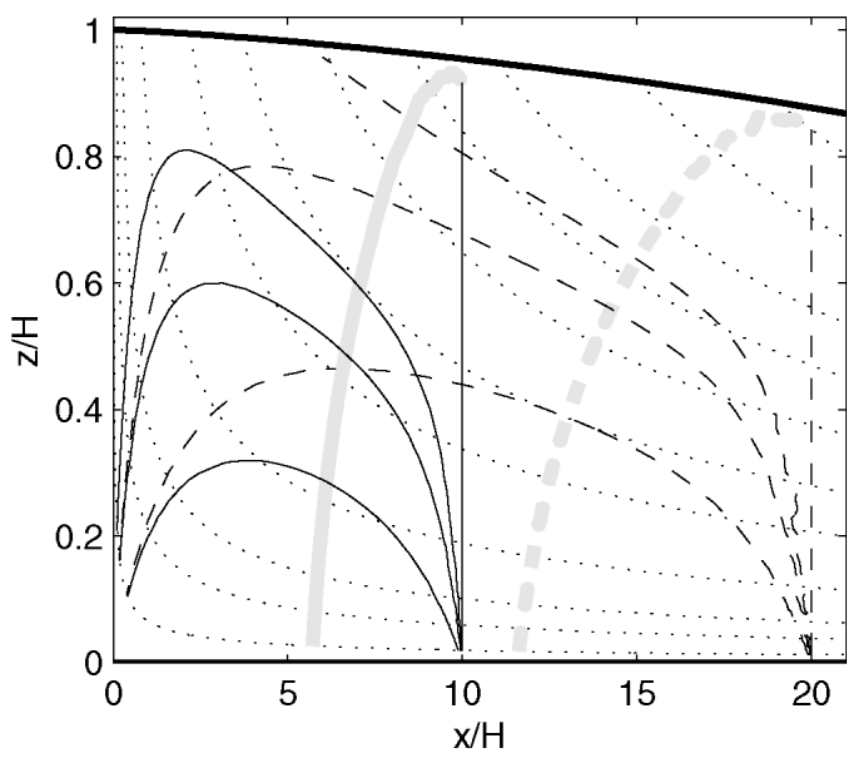

Fig. 12. Pre-core slope-angle contours $\left(\theta_{\mathrm{f}}=10^{\circ}, 30^{\circ}\right.$ and $50^{\circ}$ ) for two core locations, $10 \mathrm{H}$ (solid lines) and $20 \mathrm{H}$ (dashed lines). The thick gray lines mark where $\theta_{\mathrm{r}}=\theta_{\mathrm{f}}$. Dotted lines are particle paths. 


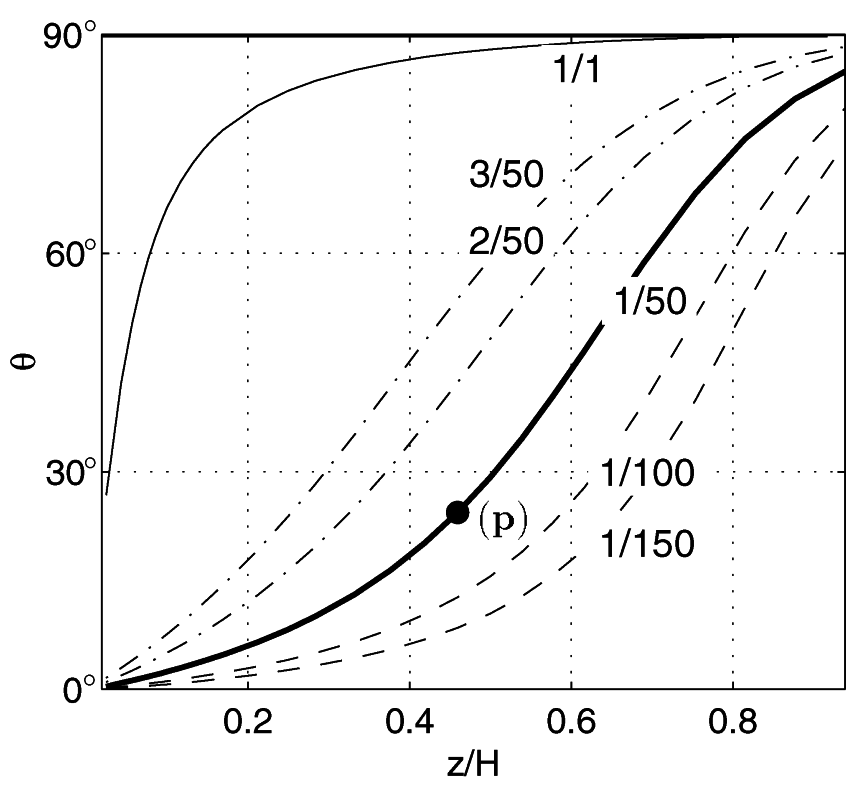

Fig. 13. The scaling of pre-core angles with variations in the $H / L$ ratio. The heavy solid line shows the angles along a vertical transect through point $(\mathbf{p})$ on Figure 6. In this case $H / L=1 / 50$ and $C / L=0.2$. The dashed lines show the equivalent angles for other $H / L$ ratios (keeping $C / L=0.2)$. The curves are related by Equation (A1).

of the modeled ice sheet is doubled while keeping $H$ the same, the pre-core slopes are halved. Since the slope is the tangent of the angle, the angles for pre-cores in two different sets of model geometries are related by

$$
\tan \left(\theta_{2}\right)=\frac{H_{2} L_{1}}{L_{2} H_{1}} \tan \left(\theta_{1}\right)
$$

Figure 13 illustrates this $H / L$ dependence of the slope angles for a vertical section through point $(\mathbf{p})$ in Figure 7.

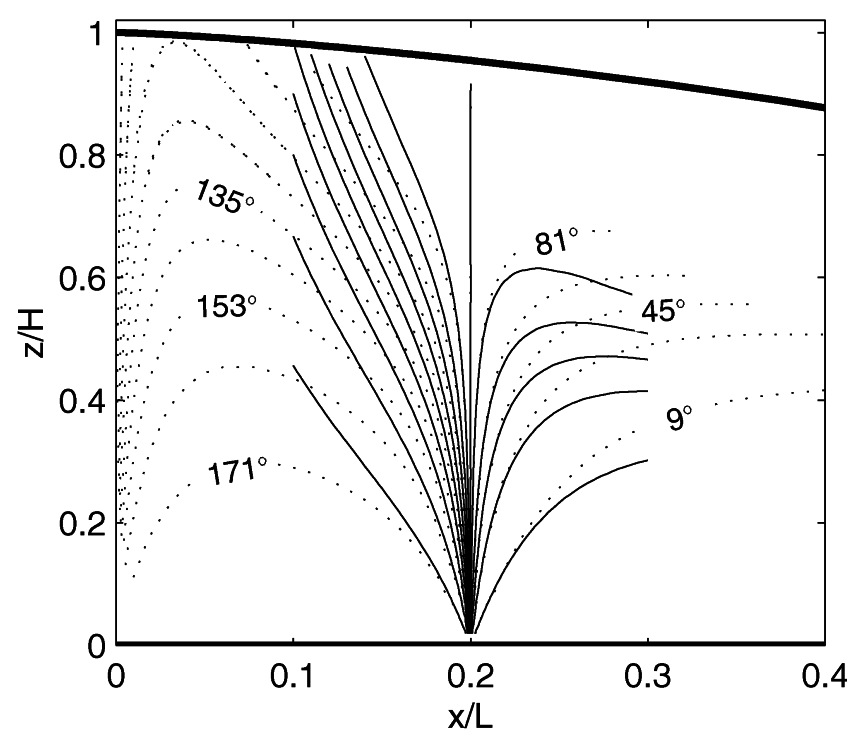

Fig. 14. Pre-core slope angles (solid lines) calculated from the "constant-strain-rate" approximation, using rates at the core location (10H). Pre-core slope contours (dotted lines) are included for comparison.
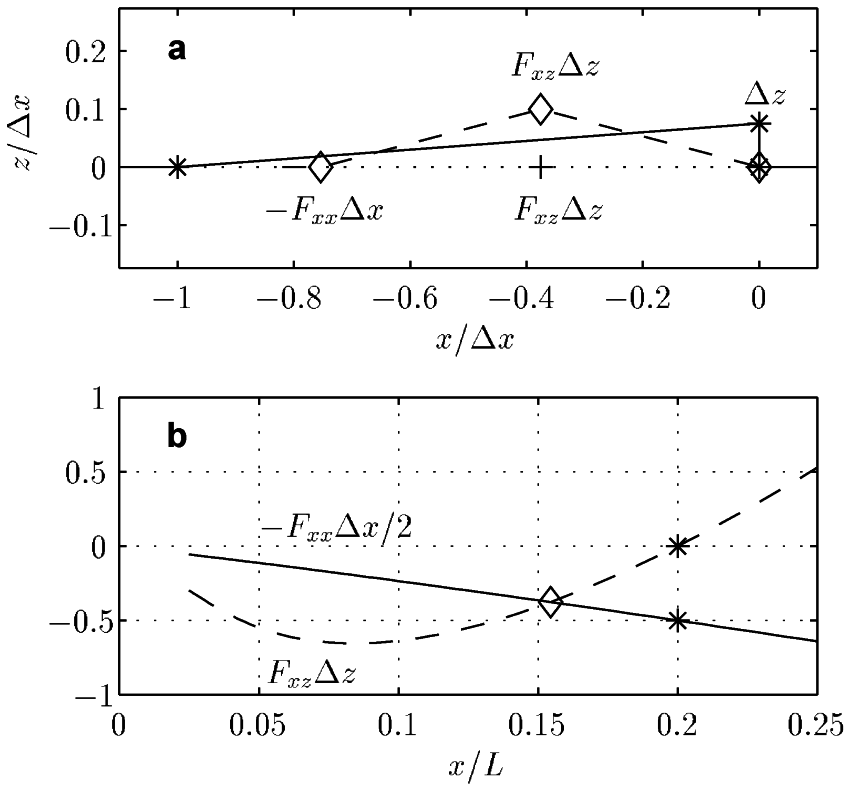

Fig. 15. (a) An overturning disturbance (*) and a possible symmetric predecessor $(\diamond)$. (b) The point at which $F_{x z} \Delta z$ equals $-F_{x x} \Delta x / 2(\diamond)$.

\section{APPENDIX B}

\section{THE APPROXIMATE LINEARITY OF $F_{x x}$ ALONG A PATH}

For our simple geometry, where $\bar{u}=\dot{b} x / h$, the position $x(t)$ along a flow path can be expressed as the exponential of an integral (using Equation (1)).

$$
\dot{x}=u=\frac{\dot{b} x}{h} \hat{u} \quad \frac{x(t)}{x(0)}=\exp \left(\int_{0}^{t} \frac{\dot{b}}{h} \hat{u} \mathrm{~d} t\right)
$$

Since $\hat{u}$ decreases along the particle path, this expression is more linear in $t$ than would be expected for a simple exponential.

The $F_{x x}$ term of $\mathbf{F}$, as a function of time along a path, can be approximated by a similar exponential. Starting with the differential equation for $\mathbf{F}$ (Equation (22)),

$$
\begin{gathered}
\dot{F}_{x x} \approx \partial_{x} u F_{x x} \quad \partial_{x} u \approx \frac{\dot{b}}{h} \hat{u} \\
F_{x x}(t) \approx \exp \left(\int_{0}^{t} \partial_{x} u \mathrm{~d} t\right) \approx \frac{x(t)}{x(0)} .
\end{gathered}
$$

Here we assume that $F_{z x}, \partial_{x} w, \partial_{x} \hat{u}$ and $\partial_{x} h$ are small, which is valid in the inner third of our ice-sheet model.

\section{APPENDIX G}

\section{HOMOGENEOUS STRAIN-RATE APPROXIMATION}

Over a short distance along a particle path, we can assume that $\mathbf{L}$ (Equation (3)) is approximately constant. It is then possible to derive an analytical expression for $\mathbf{F}$ as a function of time (Ramberg, 1975, equations 33 and 38). In the 
neighborhood of the core, the equations are $(t=0$ at the core; again using Equation (22))

$$
\begin{gathered}
\mathbf{L}_{(\mathbf{r})}=\left[\begin{array}{cc}
\partial_{x} u & \partial_{z} u \\
\partial_{x} w & \partial_{z} w
\end{array}\right] \approx\left[\begin{array}{cc}
\epsilon & \tau \\
0 & -\epsilon
\end{array}\right] \\
\mathbf{F}(t) \approx\left[\begin{array}{cc}
\mathrm{e}^{\epsilon t} & \frac{\tau}{2 \epsilon}\left(\mathrm{e}^{\epsilon t}-\mathrm{e}^{-\epsilon t}\right) \\
0 & \mathrm{e}^{-\epsilon t}
\end{array}\right] \\
\tan \theta_{\mathrm{f}} \approx \frac{F_{z z}}{-F_{x z}} \approx \frac{\epsilon}{\tau} \frac{-2}{\mathrm{e}^{2 \epsilon t}-1} .
\end{gathered}
$$

As seen in Figure 14, this approximation captures the rapid rotation of the pre-core slope near the core. Further away, the variation in the velocity gradient must be taken into account. A similar approximation is used in Waddington and others (2001) to estimate segment overturn times.

\section{APPENDIX D}

\section{THE ORIGIN OF A SYMMETRIGAL DISTURBANGE}

Consider a fold with a vertical leading edge at point (q). If its amplitude is $\Delta z$, and width is $\Delta x$, then its trailing-limb slope is $\Delta z / \Delta x$. We can approximate it by a right triangle with a set of points with relative positions (Fig. 15a)

$$
\left\{\left[\begin{array}{l}
0 \\
0
\end{array}\right],\left[\begin{array}{c}
0 \\
\Delta z
\end{array}\right],\left[\begin{array}{c}
-\Delta x \\
0
\end{array}\right]\right\} .
$$

The relative positions at an earlier time would be (using Equation (19))

$$
\left\{\left[\begin{array}{l}
0 \\
0
\end{array}\right],\left[\begin{array}{l}
F_{x z} \Delta_{z} \\
F_{z z} \Delta z
\end{array}\right],\left[\begin{array}{l}
-F_{x x} \Delta x \\
-F_{z x} \Delta_{x}
\end{array}\right]\right\}
$$

where the $\mathbf{F}$ reference point is $(\mathbf{q})$. Here we assume that $-F_{z x} \Delta x \approx 0$. When $F_{x z} \Delta z=-F_{x x} \Delta x / 2$ as shown in Figure $15 \mathrm{~b}$, the earlier disturbance is a symmetrical triangle with height $F_{z z} \Delta z$. For smaller trailing $\Delta z / \Delta x$ at the core, the symmetric injection time is earlier. For example, in Figure $6(\mathbf{q})$, this slope is 0.04 and we would infer that the disturbance was injected at $x / L \approx 0.12$. However, in Figure 15, where the fold is observed with $\Delta z / \Delta x=0.075$, the symmetric injection point is closer to the core site $(x / L=0.16)$. It is possible, if $\Delta z / \Delta x$ is too small, that no point satisfies this constraint. In this case, the observed fold could not have originated as a symmetric wrinkle. 